PALABRAS CLAVE

Concentración económica Grandes empresas

Activos de capital

Inversiones

Indicadores económicos

Análisis de regresión

Crecimiento económico

México
Germán Alarco

Investigador Principal y Profesor del

Centro de Negocios (CENTRUM)

de la Pontificia Universidad

Católica del Perú

o galarco@pucp.edu.pe

Patricia del Hierro

Profesora de la Universidad de Piura,

Campus Lima

œpathierro@hotmail.com
REVISTA CEPAL 101 • AGOSTO 2010

\section{Crecimiento y concentración de los principales grupos empresariales en México}

\author{
Germán Alarco y Patricia del Hierro
}

$\mathrm{E}$

n este trabajo se discuten diversas hipótesis sobre el origen y operación de los grupos económicos y se plantea un modelo destinado a explicar las fuentes de crecimiento de los activos totales. Se muestra la creciente contribución de los grupos económicos mexicanos al PIB, mientras que sus aportaciones en términos del empleo y las utilidades son menores. Se observa a lo largo del tiempo un claro fenómeno de concentración de las ventas y activos en favor de los grupos más importantes. Se concluye que la principal fuente de financiamiento de la expansión de los activos entre 2005 y 2007 fueron el crecimiento de los pasivos y, en segundo lugar, las aportaciones patrimoniales de los accionistas. Se determina que la inversión de los principales grupos se realiza de manera discreta en el tiempo y que estos tienden a "sobreinvertir" como una forma de obstruir la entrada de otros competidores. 


\section{I}

\section{Introducción}

La discusión en torno de la presencia de los grupos económicos empresariales es antigua e importante, ya que en algunos países no solo tienen una participación relevante en la producción, la inversión y el empleo, sino que fueron los actores principales de los procesos de industrialización de muchas economías en particular. El retorno, más reciente, a estructuras empresariales más especializadas y menos diversificadas no ha reducido su preponderancia a nivel internacional. La visión acerca de los grupos coexiste también con quienes piensan que estos reflejan distorsiones o fallas en los mercados que pueden afectar a los procesos competitivos.

El estudio de la empresa y de los grupos empresariales en México no es reciente. Basave y Hernández (2007, pp. 94-119) presentan una reseña de su evolución, con los trabajos pioneros de Cossío Villegas y Ceceña en los años setenta, donde el énfasis estuvo en la industrialización y los monopolios. Posteriormente, en el marco de la estrategia de sustitución de importaciones y de una economía cerrada, la orientación se dirigió al análisis de la concentración en los sectores-ramas económicas y en el rol de la inversión extranjera directa. En los años ochenta, se tendía a explicar el desarrollo industrial a partir de la influencia del capital financiero (antes y después de la crisis de 1982). Más recientemente, el enfoque se propone explicar la transnacionalización y la dinámica exportadora de las grandes empresas mexicanas.

En el mundo real, los grupos económicos latinoamericanos, incluidos los mexicanos, surgieron en tres períodos (Mortimore y Peres, 2001, p. 51): desde fines del siglo XIX, durante la primera oleada de industrialización del país, por intermedio de grupos de empresarios que crearon grandes fábricas manufactureras no integradas formalmente entre sí y bancos para financiarlas; en la segunda oleada de la industrialización, iniciada en los años treinta del siglo pasado, se pasó a una red con empresas agrupadas a través de controladoras (holding) (Chavarín, 2006, pp. 195-196). En la tercera surgen los nuevos grupos económicos vinculados a la reestructuración productiva neoliberal — caracterizada por la privatización,

$\square$ Los autores agradecen el apoyo de Malcolm Stewart Robles como asistente general del estudio y a los dictaminadores anónimos que comentaron el documento. la desregulación y la globalización — iniciada a partir de los años ochenta (Fernández, 2000, p. 97), donde destacan (de los 10 primeros grupos mexicanos) tres grandes grupos: Slim, Zambrano-Cementos Mexicanos (Cemex) y Salinas Pliego-Elektra.

El mapa de las grandes empresas ubicadas en México comprende a las empresas transnacionales, el fortalecimiento y modernización de los grupos tradicionales y la emergencia de nuevos y muy poderosos conglomerados formados desde los años ochenta. Las empresas nacionales aumentaron aceleradamente de tamaño y evolucionaron hacia una estructura transnacionalizada de tamaño medio, fruto de la exportación de productos y capitales, mientras que la propiedad se mantuvo en los perfiles tradicionales (Garrido, 1997, pp. 8-9). La defensa ante la apertura comercial y el Tratado de Libre Comercio de América del Norte, en el caso de las empresas orientadas al mercado interno, condujo a que se establecieran alianzas estratégicas con las empresas extranjeras, se incrementaran las barreras a la entrada (inversiones preventivas) y se combinaran el uso del poder sobre esos mercados y las relaciones políticas (Garrido, 2001, pp. 2-3).

Los objetivos de este trabajo son múltiples. En primer lugar, presentar una pequeña reseña sobre los elementos que pueden dar cuenta del origen y mantenimiento de los grupos económicos; asimismo, se propone un modelo para explicar las fuentes de crecimiento de los activos totales. En segundo lugar, mostrar la información estadística y su procesamiento para determinar la importancia de los principales grupos de la economía mexicana en términos del producto interno bruto (PIB), el empleo y la generación de excedentes. En tercer lugar, explorar la dinámica de crecimiento de los grupos económicos mexicanos entre 2004 y 2007 sobre la base del modelo desarrollado en la primera parte y de una ecuación de corte transversal.

El artículo comprende tres secciones y las reflexiones finales. En la primera parte se incorpora una reseña en que se evalúan los conceptos de diversificación, sinergia, redes, diversas hipótesis sobre el surgimiento y operación de los grupos económicos y un modelo simple para explicar las fuentes de crecimiento en los activos de esos grupos. En la segunda parte, se señala la relevancia de estos grupos en la economía mexicana y los niveles de concentración, en su interior, de los 
activos, pasivos y patrimonio. En la tercera sección se exploran las fuentes de crecimiento de estos grupos en el período bajo análisis. Finalmente, se presentan dos anexos con la información utilizada en el análisis de regresión.

En este artículo no se aborda el análisis de las empresas transnacionales y de los empresarios medianos y pequeños con los que comparten espacio los empresarios más importantes de la economía mexicana. Se asume que los principales empresarios mexicanos actúan bajo la modalidad de grupo económico. No se examina la historia de los grandes grupos económicos mexicanos y sus relaciones con el Estado, ni se analiza el comportamiento específico de algunos de ellos en tiempos de auge (Garrido, 2002; Castañeda, 2004) o de crisis (Garrido, 2001).

\section{II}

\section{Diversificación, contribución y posibilidades de crecimiento de los grupos económicos}

El tema fundamental para definir a los grupos económicos es la diversificación en los negocios. Se discute el concepto y las formas de medirla, pero lo más tradicional es concebirla como la participación activa en varios negocios distintos relacionados o no (Huerta y Navas, 2007, pp. 134-135). La siguiente materia a considerar es la relación entre diversificación y resultados en los negocios. Al respecto, estos mismos autores sostienen que, no obstante los numerosos estudios realizados, la vinculación entre diversificación y resultados permanece inconclusa. Sin embargo infieren, por una parte, que las empresas diversificadas presentan resultados superiores en relación con las empresas de negocio único, ceteris paribus y, por otra, que las empresas cuya diversificación es relacionada presentan resultados superiores con respecto a las empresas que muestran una diversificación no relacionada (ibíd., pp. 138 y 141).

Los conceptos de sinergia y redes son fundamentales para entender a los grupos económicos. En el primero de los casos, Huerta y Navas (2007, pp. 137-138) señalan que los recursos relacionados entre los negocios son fuente de sinergias (el todo es mayor que la simple suma de las partes) que mejoran el valor corporativo del conjunto. Por otra parte, las redes formales e informales son una vía para abatir los costos de transacción en el mercado mediante la selección de recursos más baratos. Estas redes también pueden ser vistas desde el enfoque social, que privilegia los contactos personales como herramienta principal para obtener los resultados deseados (Levanti, 2001, p. 1.046).

Los grupos económicos deben entenderse como una forma de red de negocios que surge de una combinación particular de arquitectura organizacional y gobierno corporativo, donde un conjunto de empresas es controlado por un pequeño número de grandes accionistas, usualmente miembros de una familia extendida o de un círculo cerrado de asociados con nexos sociales. Las transacciones que tienen lugar entre las empresas de un grupo suelen sostenerse en el largo plazo y responden a deficiencias institucionales, por ejemplo, el funcionamiento o la formación de ciertos mercados como el crediticio, la disponibilidad de ciertos trabajadores o de algunos insumos, la presencia de fallas de mercado u otras explicaciones de la economía política, sociológicas, culturalistas, entre otras (Chavarín, 2006, pp. 194 y 195).

El enfoque de las fallas o de las imperfecciones del mercado señala que los grupos económicos suplen las dificultades para obtener capital, mano de obra especializada, materias primas, componentes y tecnología en las economías emergentes (Guillén, 2000, p. 363). En la misma dirección, Rendón (1997, p. 5) plantea que los grupos surgen ante la incertidumbre de garantizar la calidad y el tiempo de entrega de insumos necesarios para producir y vender.

En la segunda aproximación, por parte de los economistas sociologistas, se señala que las empresas replican la estructura social que las rodea, de tal forma que los patrones de autoridad vertical, horizontal y recíproca afectan a la organización de la firma y a las relaciones entre las empresas. Estos patrones de autoridad se asumen como relativamente estables en el tiempo y resistentes a las presiones externas. Los ejemplos más característicos se ubican en la República de Corea (estructura vertical), la provincia china de Taiwán (horizontal) y el Japón (recíprocos). En la tercera aproximación, en el caso de los países de industrialización reciente, se vincula el crecimiento de los grupos económicos a las políticas a 
cargo del Estado, los bancos o de ambos para promover el desarrollo económico. El Estado prefiere tratar con pocos empresarios como agentes del sector privado (Guillén, 2000, pp. 363 y 364).

Otra modalidad de visualizar las teorías para explicar el surgimiento de los grupos económicos es la propuesta por Tarziján y Paredes (2006, pp. 56-58), quienes plantean que la estructura de los grupos económicos respondió en su tiempo a los incentivos económicos de la región, y que al cambiar las instituciones que dieron lugar a esos incentivos, esa estructura organizacional es hoy menos útil y ha variado. Asimismo, la hipótesis que sostienen dichos autores es que los grupos económicos se forman y fortalecen para generar una estructura y red de influencias que les permita acceder al poder político y, de este modo, potenciar su capacidad y accionar económico.

Khanna y Yafeh (2007, pp. 333, 336-341) proponen y analizan seis hipótesis acerca de las razones que existen para la formación de los grupos económicos, su permanencia en diferentes contextos y algunas implicaciones sobre el bienestar. En la primera hipótesis se señala que los grupos económicos diversificados son más comunes en economías con instituciones de mercado menos desarrolladas. Al respecto, luego de una extensa revisión bibliográfica, concluyen que el escaso desarrollo de los mercados de capitales no es el elemento determinante en la formación de un grupo empresarial. Al parecer son otro tipo de instituciones y situaciones las que los favorecen, tales como las leyes laborales poco claras, el escaso desarrollo de las habilidades de los trabajadores y los gerentes o el objetivo de pagar menos impuestos. Tampoco es nítida la correlación entre mayor diversificación y mayores beneficios, pues no existe una correlación lineal. En general, los resultados son ambiguos, como en el caso chileno - con un buen desarrollo de los mercados de capitales y reglas laborales más o menos claras-donde se ha intensificado la formación de grupos que se han propuesto la diversificación.

En la segunda hipótesis desarrollada por Khanna y Yafeh (2007, pp. 341-343) se argumenta que la formación de grupos económicos, por medio de la integración vertical y el volumen de intercambio intragrupos, tiende a ser mayor cuando existe un magro desarrollo de las instituciones legales y judiciales, que elevan los costos de contratación. La evidencia no avala esta hipótesis y pareciera que la integración vertical tiene más que ver con el deseo de la empresa de aumentar su poder monopólico. En la tercera hipótesis se sostiene que la formación de grupos piramidales (cuyo control está en manos de pocos accionistas) es común en países donde la ley protege muy débilmente a los inversionistas. A su vez, esto obliga a los inversionistas a solicitar un descuento cuando compran acciones en una empresa perteneciente a un grupo económico.

Con relación a esta hipótesis no se encontró evidencia alguna. Los grupos piramidales tienden a formarse en los casos en que el Estado ha implementado leyes de apoyo o protección a las industrias, por ejemplo, los Chaebol de la República de Corea. De igual modo, esa conformación grupal se aviene más con el propósito de aumentar la estabilidad de las ganancias que de maximizarlas. Además, a través de estos grupos procuran transferir entre sí los apoyos o subsidios que reciben del Estado. Estas estructuras grupales les permiten también excederse en la inversión a fin de establecer defensas o barreras respecto de otros competidores internos o externos.

En la cuarta hipótesis se afirma que el control familiar de los grupos económicos es más común en países cuyas reglas son inadecuadas y donde las transacciones con terceros son costosas. Asimismo, estos grupos continúan existiendo por razones societales, más allá de las vinculaciones familiares. $\mathrm{Al}$ respecto, existe una mayor presencia familiar en los países con instituciones y mercados poco desarrollados. Por ejemplo, en Singapur y Malasia se encontró que 9 de 10 y 35 de 50 grupos tenían gerentes que pertenecían, respectivamente, a las familias que ejercen el control de las empresas. De igual modo, existen factores sociales, culturales, institucionales y otros que van más allá de la lógica puramente económica, que determinan e influyen en la creación, preservación y operación de los grupos económicos (Khanna y Yafeh, 2007, pp. 348-351).

En la quinta hipótesis se plantea que la creación, expansión y diversificación de los grupos económicos se produce como resultado del apoyo gubernamental, aunque su rentabilidad depende de las habilidades y oportunidades que logren aprovechar. Así, a partir de la evidencia, se encontró que los grupos económicos de muchos países se formaron gracias al apoyo deliberado del gobierno. Sin embargo, aunque se logró confirmar que ese apoyo es muy importante para la formación de grupos empresariales, en otros países también surgieron grupos con escasa o nula ayuda gubernamental. En general, lo que se observa es que en su comienzo la gran mayoría de los grupos empresariales están integrados por familias con vínculos muy cercanos al gobierno de turno.

En virtud de su propia dinámica los grupos económicos acumulan influencia y poder tanto económico como político. Esto redunda en que su relación con el ámbito estatal se vuelva fluctuante, ambigua y cambiante, lo que 
en ocasiones provoca la reacción del Estado que trata de reducir la influencia de esos grupos económicos, de modo que sus representantes tienen que realizar intensas negociaciones (lobby) para no perder el favor de las autoridades. En determinadas circunstancias los grupos también se enfrentan al Estado, cuando tratan de resistir regulaciones antimonopólicas y otras similares. Khanna y Yafeh (2007, pp. 352-361) descubren una fructífera relación entre el Estado y los grupos empresariales, que también tiene sus retrocesos, puesto que se trata de un juego entre ambas partes y los juegos son típicamente complejos. Si bien cohabitan, su coexistencia no resulta fácil.

La última hipótesis de Khanna y Yafeh (2007, pp. 361-362) se refiere a que los grupos económicos realizan una serie de prácticas no competitivas que tienden a incrementar el poder de mercado, y este mayor poder monopólico se reflejaría en altas tasas de beneficios, más aún cuando estas pueden establecer barreras al comercio y existe un débil marco regulatorio en favor de la competencia. Sobre el particular, no queda del todo claro si al aumentar el grado de monopolio existe una tasa de ganancia mayor. Se observa que en entornos tan variables no se crean condiciones estables que permitan garantizar un grado de monopolio determinado y ganancias abundantes. Quizás esto explica por qué los grupos empresariales siempre están tratando de influir en las decisiones del Estado.

Luego de la revisión bibliográfica, se ha diseñado un modelo para explicar las fuentes de expansión de los grupos económicos a partir de la descomposición entre el financiamiento interno (utilidades retenidas) y externo. Se parte del balance general de estos grupos, donde la diferencia interanual de los activos totales $(A)$ de un año determinado respecto del anterior es igual a la suma de las variaciones interanuales de los pasivos $(L)$ y del patrimonio $(E)$. En los activos totales se consideran los activos financieros (incluidas las inversiones), activos fijos y otros activos, ya que la expansión de un grupo puede realizarse por la vía de diferentes canales. En la ecuación (2) se expresan estos cambios en términos discretos, donde el crecimiento de los activos podría financiarse mediante deuda o capital propio.

$$
\begin{gathered}
A_{t}-A_{t-1}=\left(L_{t}-L_{t}-1\right)+\left(E_{t}-E_{t-1}\right) \\
\Delta A=\Delta L+\Delta E
\end{gathered}
$$

No se discutirán aquí los límites al financiamiento externo, pero es claro que este es posible en tanto mantiene proporciones determinadas con el crecimiento del capital propio. Normalmente, ningún grupo va a poder endeudarse por sobre los estándares de la actividad que tradicionalmente se relacionan con una proporción determinada de los aportes de los socios del grupo económico. El crecimiento del patrimonio puede explicarse por las utilidades del ejercicio $(P)$ y las ampliaciones de capital directas o a través de los mercados de valores $(N S)$. El endeudamiento externo al grupo se establecería como una proporción del incremento del capital propio, considerando dos razones vinculadas al total de pasivos respecto de los activos totales $(\alpha)$ y otra del activo respecto del patrimonio $(\beta)$ de las ecuaciones (3) y (4), que se reemplazarían en la ecuación (2) para obtener la (5).

$$
\begin{gathered}
\frac{L}{A}=\alpha \\
\frac{A}{E}=\beta \\
\Delta A=(1+\alpha \beta)(N S+P)
\end{gathered}
$$

Las utilidades pueden explicarse por diferentes variables tradicionales, como el producto del rendimiento sobre el patrimonio (roe) por el patrimonio de la ecuación (6) o como el rendimiento sobre las ventas (ros) por las ventas totales $(S)$, siendo estas últimas el producto de la participación de las ventas del grupo económico en la actividad $(\chi)$, de las ventas de la actividad en el producto $(\delta)$ por el PIB $(Y)$ que se muestra en la ecuación (7). No se distingue aquí entre utilidades y utilidades retenidas después de dividendos.

$$
\begin{gathered}
\Delta A=(1+\alpha \beta)(N S+\text { roe } E) \\
\Delta A=(1+\alpha \beta)[N S+\operatorname{ros}(\chi \delta Y)]
\end{gathered}
$$

El crecimiento de los activos totales de cualquier grupo económico se explicaría, en relación directa, por el crecimiento del producto, ${ }^{1}$ las posibilidades de ampliar el patrimonio con los mismos socios, socios nuevos (o ambos) o a través de mercados de valores. También inciden en el crecimiento de los activos, de manera directa, las razones pasivos/activos totales, activos totales/patrimonio, del rendimiento sobre las ventas del grupo económico, y la participación de la empresa y de la actividad en la economía.

\footnotetext{
${ }^{1}$ No se están considerando, por simplicidad, las ventas externas que dependerían de la dinámica del producto o servicio a nivel internacional y de su competitividad externa.
} 


\section{III \\ Participación de los grupos económicos en la economía mexicana}

La información estadística disponible acerca de los grupos económicos nacionales proviene de la revista Expansión, que publica todos los años, entre finales de abril y junio, un reportaje titulado Los 100 empresarios más importantes de México. En él se entrega en orden descendente información sobre los empresarios nacionales y las empresas bajo su control. Aquí se ha seleccionado la información relativa a los cuatro últimos años de 2004 a 2007, publicada entre 2005 y 2008, ya que es más homogénea. ${ }^{2}$ Estos empresarios de mayor gravitación, que se encuentran en diversas actividades económicas, generalmente no actúan de manera aislada $\mathrm{y}$ forman parte de grandes organizaciones que operan como grupos económicos (Rendón y Morales, 2008b, p. 1.184).

Para cada grupo económico se presentan las ventas, la capitalización bursátil, el volumen operado en la Bolsa de Valores de México, el número de trabajadores, la utilidad neta, el rendimiento sobre el patrimonio (roe), la relación precio de la acción respecto de la utilidad por acción al final de diciembre de cada año y la relación activo/deuda. En forma complementaria se muestra la participación de mercado y el índice de poder económico elaborado por la revista Expansión. Solo en el primer año (2004) se agregan los registros sobre el total de activos y del patrimonio.

Con la información parcial de los años 2004, 2005, 2006 y 2007 proporcionada por Expansión, se ha procedido a estimar los activos totales, pasivos y patrimonio de cada uno y de todos los grupos. La estimación del patrimonio $(E)$ se realiza a partir del conocimiento de la utilidad neta del ejercicio $(P)$ y de la tasa de rendimiento sobre el patrimonio (roe) que se muestra en la ecuación (8). El total de pasivos $(L)$ se determina a partir de la identidad contable, que señala que los activos totales son iguales al pasivo más el patrimonio, tal como se indica en la ecuación (9). Al dividir cada lado de esta expresión por $(L)$, se obtiene la relación activos totales sobre pasivos, según se muestra en la ecuación (10).

\footnotetext{
${ }^{2}$ La información consignada por Expansión sobre los 100 empresarios mexicanos es de mayor antigüedad, pero lamentablemente diferenciada a través del tiempo.
}

$$
\begin{aligned}
& \text { roe }=\frac{P}{E} \\
& A=L+E \\
& \frac{A}{L}=1+\frac{E}{L}
\end{aligned}
$$

Luego de obtener los resultados mediante el procedimiento anterior, se detectaron algunas inconsistencias en la información. El valor de los activos totales, los pasivos y el patrimonio mostró, en el caso de algunos empresarios, marcadas modificaciones entre 2006 y 2007. Se detectó que las inconsistencias ocurrían cuando los rendimientos sobre el patrimonio se modificaban de manera importante sin correspondencia alguna con respecto a lo que ocurría con las utilidades netas. El caso del primer grupo mexicano (Slim) es ilustrativo, ya que el rendimiento sobre el patrimonio (roe) se redujo de $26,4 \%$ a $12,6 \%$ entre 2006 y 2007 , mientras que las utilidades se elevaron de 77.450 millones a 122.370 millones de pesos y la relación activo total/pasivos se mantuvo constante en 1,6 veces.

Para corregir este problema se evaluaron diferentes alternativas considerando rangos en las variaciones en las ventas, en el roe, en la utilidad neta y en la relación activos totales/deuda. Estas se evaluaron conceptualmente y luego se aplicaron a la base de datos. Sin embargo, finalmente se adoptó un nuevo criterio mediante el cual el roe del año 2007 sería igual al del año anterior (2006) multiplicado por la utilidad de 2007 y dividido por la utilidad de 2006. De esta forma se evitarían las variaciones bruscas en el patrimonio, y de ahí en los pasivos y en los activos totales. Por otra parte, para garantizar la consistencia de la información, se dispuso eliminar toda la información del empresario o grupo económico para un año determinado si es que faltaban los datos concernientes a los activos totales, a los pasivos o al patrimonio. Mantener a ese empresario hubiera significado afectar al equilibrio de la sumatoria de los balances generales (o del estado de situación financiera).

En el cuadro 1 se puede apreciar la contribución al PIB de los 100 empresarios más importantes de México, del empleo asalariado, de la población ocupada y del excedente de explotación en los años bajo análisis. Para 
CUADRO 1

Contribución de los grupos económicos al producto, empleo y excedente de explotación

(En porcentajes)

\begin{tabular}{|c|c|c|c|c|c|}
\hline \multirow{2}{*}{ Grupos } & \multirow{2}{*}{ Año } & \multirow{2}{*}{$\begin{array}{c}\text { Producto } \\
\text { interno bruto }\end{array}$} & \multicolumn{2}{|c|}{ Empleo } & \multirow{2}{*}{$\begin{array}{c}\text { Excedente } \\
\text { de explotación }\end{array}$} \\
\hline & & & Asalariados & Población ocupada & \\
\hline \multirow{4}{*}{ Grupos del 1 al 5} & 2004 & 11,87 & 2,05 & 1,17 & 2,12 \\
\hline & 2005 & 9,60 & 1,70 & 1,00 & 2,03 \\
\hline & 2006 & 10,09 & 1,68 & 1,01 & 2,08 \\
\hline & 2007 & 10,65 & 1,78 & 1,08 & $\ldots$ \\
\hline \multirow[t]{4}{*}{ Grupos del 6 al 10} & 2004 & 3,47 & 0,68 & 0,39 & 0,50 \\
\hline & 2005 & 2,77 & 0,95 & 0,56 & 0,47 \\
\hline & 2006 & 2,51 & 0,73 & 0,44 & 0,44 \\
\hline & 2007 & 3,48 & 0,97 & 0,59 & $\ldots$ \\
\hline \multirow[t]{4}{*}{ Grupos del 1 al 10} & 2004 & 15,34 & 2,73 & 1,56 & 2,62 \\
\hline & 2005 & 12,37 & 2,66 & 1,56 & 2,50 \\
\hline & 2006 & 12,59 & 2,42 & 1,45 & 2,51 \\
\hline & 2007 & 14,13 & 2,75 & 1,66 & $\ldots$ \\
\hline \multirow[t]{4}{*}{ Grupos del 11 al 20} & 2004 & 3,32 & 0,97 & 0,55 & 0,44 \\
\hline & 2005 & 3,08 & 0,89 & 0,52 & 0,47 \\
\hline & 2006 & 4,09 & 1,24 & 0,75 & 0,52 \\
\hline & 2007 & 3,36 & 1,16 & 0,70 & $\ldots$ \\
\hline \multirow[t]{4}{*}{ Grupos del 21 al 30} & 2004 & 1,57 & 0,64 & 0,37 & 0,08 \\
\hline & 2005 & 2,10 & 0,66 & 0,39 & 0,20 \\
\hline & 2006 & 2,03 & 0,68 & 0,41 & 0,19 \\
\hline & 2007 & 2,17 & 0,72 & 0,44 & $\ldots$ \\
\hline \multirow[t]{4}{*}{ Grupos del 31 al 40} & 2004 & 1,21 & 0,38 & 0,22 & 0,09 \\
\hline & 2005 & 1,57 & 0,46 & 0,27 & 0,12 \\
\hline & 2006 & 0,99 & 0,35 & 0,21 & 0,11 \\
\hline & 2007 & 1,27 & 0,36 & 0,22 & $\ldots$ \\
\hline \multirow[t]{4}{*}{ Grupos del 41 al 50} & 2004 & 1,18 & 0,29 & 0,17 & 0,07 \\
\hline & 2005 & 0,40 & 0,20 & 0,12 & 0,08 \\
\hline & 2006 & 0,78 & 0,33 & 0,20 & 0,07 \\
\hline & 2007 & 0,51 & 0,21 & 0,13 & $\cdots$ \\
\hline \multirow[t]{4}{*}{ Grupos del 51 al 100} & 2004 & 1,51 & 0,47 & 0,27 & 0,03 \\
\hline & 2005 & 1,49 & 0,51 & 0,30 & 0,12 \\
\hline & 2006 & 1,58 & 0,53 & 0,32 & 0,11 \\
\hline & 2007 & 1,49 & 0,61 & 0,37 & $\ldots$ \\
\hline \multirow[t]{4}{*}{ Total } & 2004 & 24,13 & 5,48 & 3,14 & 3,32 \\
\hline & 2005 & 21,01 & 5,39 & 3,16 & 3,49 \\
\hline & 2006 & 22,06 & 5,54 & 3,33 & 3,51 \\
\hline & 2007 & 22,91 & 5,81 & 3,51 & $\ldots$ \\
\hline
\end{tabular}

Fuente: elaboración propia sobre la base datos obtenidos de la revista Expansión y del Instituto Nacional de Estadística, Geografía e Informática (INEGI).

analizar el grado de concentración o de dispersión se presenta también la información para los 5 primeros grupos (del 6 al 10), los grupos del 11 al 20, del 21 al 30, del 31 al 40, del 41 al 50 y del 51 al 100 .

Desde 2005 a 2007, las ventas totales del conjunto de los empresarios respecto del producto fueron crecientes, con un valor equivalente al 22,9\% del PIB en 2007. No obstante, esta participación fue menor a la observada en 2004. Es interesante anotar que solo los 5 primeros grupos explicaron el 10,7\% del PIB. Los 10 primeros grupos empresariales llegaron a explicar el $14,1 \%$ del PIB en 2007, mientras que los grupos del 11 al 20 contribuyeron con un $3,4 \%$; del 21 al 30 con un $2,2 \%$; del 31 al 40 con un 1,3\%; del 41 al 50 con un $0,5 \%$; y los del 51 al 100 con un 1,5\% del PIB. Entre 2005 y 2007 se observa una mayor concentración de la producción nacional en manos de los grupos nacionales en general, aunque en mayor medida en los 10 primeros grupos. 
Conviene señalar que, en términos macroeconómicos, en el período bajo análisis se observó una tasa de crecimiento del PIB entre $3 \%$ y 3,5\% anual, bastante cercana al promedio histórico de largo plazo, pese a que los precios del petróleo iban en una tendencia alcista. Las tasas de inflación fluctuaron entre 5,2\% y 3,3\% anual, mientras que la paridad cambiaria con respecto al dólar estadounidense fue relativamente estable. El poder de compra de los perceptores de remuneraciones, el sector externo, el financiero y las finanzas públicas mantuvieron las tendencias de los años anteriores.

La contribución al empleo nacional de los 100 empresarios es relevante en términos absolutos, ya que asciende a poco más de 1,5 millones de empleos permanentes en 2007. Si estos resultados se expresan en relación con la población asalariada (que recibe sueldos y salarios) y con la población ocupada nacional, equivalentes a 25,9 y 42,9 millones de personas, respectivamente, las aportaciones no son significativas, ya que equivalen a un $5,8 \%$ y a un $3,5 \%$ del total nacional de esas poblaciones.

La contribución al empleo es menos concentrada en comparación con las ventas totales respecto del producto, ya que en 2007 los 10 primeros grupos absorbieron solo un $2,8 \%$ del empleo asalariado nacional y un $1,7 \%$ de la población ocupada total. Los cinco primeros grupos captaron un $1,8 \%$ del empleo asalariado y un $1,1 \%$ de la población ocupada total. Los grupos del 11 al 20 respondieron por un $1,2 \%$ y un $0,7 \%$, respectivamente, mientras que los grupos del 21 al 30 ocuparon en el mismo año a un $0,7 \%$ y $0,4 \%$, respectivamente.

En el caso de la contribución al excedente de explotación, esta se mide a partir de la razón de la suma de las utilidades netas totales de las empresas seleccionadas entre el excedente de explotación reportado en las cuentas nacionales. A la fecha no hay información disponible sobre el excedente para el año 2007. Sin embargo, entre 2004 y 2006 la contribución de estas empresas se elevó del 3,3\% al 3,5\% del excedente de explotación nacional. En este caso, llama la atención que si bien los 10 primeros grupos contribuyen al 2,5\% del excedente nacional, en 2004 contribuían al 2,6\% de este.

Respecto de otras fuentes de información, Rendón y Morales (2008b, pp. 1.178 y 1.180-1.181) señalaron que existe un evidente proceso de concentración en favor de las empresas y grupos económicos de mayor tamaño. Entre 1993 y 2003, las grandes empresas emplearon al $21,1 \%$ y el $28,4 \%$, respectivamente, del personal ocupado de la economía. Las 500 mayores empresas mexicanas reportadas por Expansión tuvieron ventas netas equivalentes al 18,2\% del PIB en 1990, al 50\% en el año 2000 y hasta el 73,5\% en 2004. En este último año, el 5,4\% de estas empresas fueron estatales, el 57,8\% privadas nacionales y el $36,8 \%$ extranjeras.

En el cuadro 2 se exponen algunas razones financieras aplicadas al conjunto de los 100 grupos empresariales, así como también a una selección por deciles de esos grupos. Las razones seleccionadas comprenden la rotación de las ventas obtenida como el cociente de las ventas totales y el valor de los activos totales. La segunda razón se refiere al producto medio por trabajador ocupado, que expresa el cociente de las ventas totales por trabajador, expresado en millones de pesos corrientes. La tercera, sujeta a las fluctuaciones de los mercados de valores, es el cociente del valor de capitalización de mercado (capitalización bursátil) de los grupos respecto de los activos totales. Luego se presentan las razones de apalancamiento de los pasivos totales en relación con los activos totales y de los pasivos totales con respecto al patrimonio. Por último se presenta la razón utilidad neta en relación con el patrimonio en porcentaje.

Para el total de los empresarios, la rotación de las ventas se incrementa entre 2004 y 2007, lo que implica un mejor aprovechamiento de los activos totales. Resalta que exista un mayor aprovechamiento de los activos totales entre los grupos del 11 al 50 respecto de lo que ocurre en los 10 primeros grupos donde esta rotación es menor. Entre estos, la rotación de los activos es mayor en los grupos que ocupan desde la posición 31 a la 50. En la menor rotación de los activos totales de los 10 primeros grupos económicos se reflejaría la existencia de una mayor capacidad instalada ociosa, derivada de niveles de "sobreinversión" cuyo objeto es mantener barreras a la entrada de competidores elevando los estándares de equipamiento. Estos empresarios invierten en exceso aumentando las barreras y tendrían la capacidad para atender con mayor celeridad los cambios imprevistos en la demanda. Esta estrategia defensiva es descubierta también por Mortimore y Peres (2001, p. 54).

El producto medio por trabajador del total de los empresarios analizados también ha aumentado, pero solo de 2005 a 2007, ya que se redujo entre 2004 y 2005. Por deciles, el mayor valor corresponde al grupo de los 10 primeros empresarios, seguidos por los grupos del 31 al 40 lugar. La razón del valor de capitalización del mercado respecto de los activos totales también ha crecido entre 2004 y 2007 como resultado del mejor desempeño del índice de precios y de las cotizaciones de la Bolsa Mexicana de Valores. Esta razón es también mayor en el caso de los 10 primeros grupos económicos y en los ubicados en las posiciones del 31 al 50.

Los indicadores de apalancamiento durante el período 2004-2007 no tienen una tendencia clara, y son menos 
CUADRO 2

Razones financieras de los grupos económicos mexicanos, 2004-2007

\begin{tabular}{|c|c|c|c|c|c|c|c|}
\hline Grupos & Año & $\begin{array}{c}\text { Ventas/activos } \\
\text { totales }\end{array}$ & $\begin{array}{c}\text { Ventas/ } \\
\text { trabajadores }^{\mathrm{a}}\end{array}$ & $\begin{array}{c}\text { Capitalización } \\
\text { bursátil/activos totales }\end{array}$ & $\begin{array}{c}\text { Pasivos/ } \\
\text { activos totales }\end{array}$ & $\begin{array}{l}\text { Pasivos/ } \\
\text { patrimonio }\end{array}$ & $\begin{array}{c}\text { Utilidad neta/ } \\
\text { patrimonio } \\
\text { (En porcentajes) }\end{array}$ \\
\hline Grupos del 1 al 10 & $\begin{array}{l}2004 \\
2005 \\
2006 \\
2007\end{array}$ & $\begin{array}{l}0,58 \\
0,66 \\
0,81 \\
0,82\end{array}$ & $\begin{array}{l}2,08 \\
1,80 \\
2,12 \\
2,21\end{array}$ & $\begin{array}{l}0,71 \\
1,07 \\
1,58 \\
1,69\end{array}$ & $\begin{array}{l}0,61 \\
0,56 \\
0,55 \\
0,62\end{array}$ & $\begin{array}{l}1,54 \\
1,28 \\
1,22 \\
1,61\end{array}$ & $\begin{array}{l}15,41 \\
18,41 \\
22,04 \\
28,86\end{array}$ \\
\hline Grupos del 11 al 20 & $\begin{array}{l}2004 \\
2005 \\
2006 \\
2007\end{array}$ & $\begin{array}{l}0,97 \\
0,68 \\
1,07 \\
1,12\end{array}$ & $\begin{array}{l}1,27 \\
1,33 \\
1,34 \\
1,25\end{array}$ & $\begin{array}{l}0,83 \\
0,41 \\
0,75 \\
1,13\end{array}$ & $\begin{array}{l}0,49 \\
0,65 \\
0,54 \\
0,57\end{array}$ & $\begin{array}{l}0,98 \\
1,86 \\
1,15 \\
1,30\end{array}$ & $\begin{array}{l}15,49 \\
17,75 \\
17,96 \\
16,70\end{array}$ \\
\hline Grupos del 21 al 30 & $\begin{array}{l}2004 \\
2005 \\
2006 \\
2007\end{array}$ & $\begin{array}{l}0,81 \\
0,95 \\
1,19 \\
1,41\end{array}$ & $\begin{array}{l}0,90 \\
1,23 \\
1,22 \\
1,29\end{array}$ & $\begin{array}{l}0,62 \\
1,01 \\
1,13 \\
1,04\end{array}$ & $\begin{array}{l}0,58 \\
0,46 \\
0,57 \\
0,57\end{array}$ & $\begin{array}{l}1,38 \\
0,86 \\
1,33 \\
1,32\end{array}$ & $\begin{array}{r}5,87 \\
9,89 \\
15,85 \\
18,74\end{array}$ \\
\hline Grupos del 31 al 40 & $\begin{array}{l}2004 \\
2005 \\
2006 \\
2007\end{array}$ & $\begin{array}{l}0,82 \\
0,91 \\
0,77 \\
1,27\end{array}$ & $\begin{array}{l}1,17 \\
1,31 \\
1,15 \\
1,50\end{array}$ & $\begin{array}{l}0,62 \\
0,43 \\
0,76 \\
1,17\end{array}$ & $\begin{array}{l}0,50 \\
0,61 \\
0,55 \\
0,55\end{array}$ & $\begin{array}{l}1,00 \\
1,54 \\
1,20 \\
1,24\end{array}$ & $\begin{array}{r}7,07 \\
10,32 \\
11,83 \\
8,88\end{array}$ \\
\hline Grupos del 41 al 50 & $\begin{array}{l}2004 \\
2005 \\
2006 \\
2007\end{array}$ & $\begin{array}{l}1,45 \\
0,43 \\
0,98 \\
0,64\end{array}$ & $\begin{array}{l}1,49 \\
0,77 \\
0,95 \\
1,02\end{array}$ & $\begin{array}{l}0,59 \\
0,33 \\
0,88 \\
1,31\end{array}$ & $\begin{array}{l}0,55 \\
0,68 \\
0,55 \\
0,43\end{array}$ & $\begin{array}{l}1,22 \\
2,16 \\
1,22 \\
0,75\end{array}$ & $\begin{array}{l}11,11 \\
16,40 \\
11,55 \\
17,29\end{array}$ \\
\hline Grupos del 51 al 100 & $\begin{array}{l}2004 \\
2005 \\
2006 \\
2007\end{array}$ & $\begin{array}{l}0,70 \\
0,57 \\
0,98 \\
0,65\end{array}$ & $\begin{array}{l}1,18 \\
1,13 \\
1,22 \\
1,06\end{array}$ & $\begin{array}{l}0,40 \\
0,28 \\
0,44 \\
0,46\end{array}$ & $\begin{array}{l}0,62 \\
0,60 \\
0,45 \\
0,58\end{array}$ & $\begin{array}{l}1,64 \\
1,53 \\
0,83 \\
1,40\end{array}$ & $\begin{array}{l}2,27 \\
6,93 \\
7,64 \\
8,77\end{array}$ \\
\hline Total & $\begin{array}{l}2004 \\
2005 \\
2006 \\
2007\end{array}$ & $\begin{array}{l}0,67 \\
0,68 \\
0,89 \\
0,88\end{array}$ & $\begin{array}{l}1,62 \\
1,51 \\
1,62 \\
1,70\end{array}$ & $\begin{array}{l}0,69 \\
0,84 \\
1,28 \\
1,45\end{array}$ & $\begin{array}{l}0,59 \\
0,58 \\
0,54 \\
0,60\end{array}$ & $\begin{array}{l}1,44 \\
1,36 \\
1,19 \\
1,48\end{array}$ & $\begin{array}{l}13,65 \\
16,16 \\
19,02 \\
23,52\end{array}$ \\
\hline
\end{tabular}

Fuente: elaboración propia sobre la base de datos obtenidos de la revista Expansión y del Instituto Nacional de Estadística, Geografía e Informática (INEGI).

a Millones de pesos corrientes por trabajador.

variables cuando se habla de la razón pasivos totales respecto de los activos totales. La otra razón de los pasivos totales respecto del patrimonio es más fluctuante. En el caso de ambas razones, estas se elevan entre los años 2006 y 2007. Los pasivos totales llegaron a representar el $60 \%$ de los activos totales y el 148\% del patrimonio para el conjunto de las empresas. Los 10 primeros grupos tienen razones un poco más elevadas equivalentes al $62 \%$ y al $161 \%$, respectivamente, en 2007.

La última razón corresponde al cociente de la utilidad neta con respecto al patrimonio. Entre 2004 y 2007 esta se eleva del 14 al 24\%. Los 10 primeros grupos tienen las mayores razones de rendimiento para todos los años, mientras que los otros deciles muestran las menores tasas de rendimiento. A mayor tamaño, los rendimientos absolutos y relativos son superiores.
En el gráfico 1 se pueden analizar los niveles de concentración de los activos totales, pasivos totales y del patrimonio de los 100 empresarios-grupos más importantes de México. Los 10 primeros grupos nacionales poseen una participación cada vez mayor en los activos totales, al explicar el $60,8 \%$ de estos en 2005 , el 62,9\% en 2006 y el 66,8\% en 2007. Los 10 primeros grupos económicos tuvieron el $59,1 \%$ de los pasivos totales en 2005, el 63,7\% en 2006 y el $69 \%$ en 2007. En el caso del patrimonio no hay una tendencia clara, ya que la participación en dichos años fluctuó entre el $63,1 \%$ en 2005 , el $61,8 \%$ en 2006 y el $63,5 \%$ en 2007.

Los elevados niveles de concentración se ratifican al estimar el índice de Herfindahl-Hirschmann (IHH) para los diferentes deciles de los 100 principales grupos empresariales de México. En este caso, el índice se define 
Distribución de activos, pasivos y patrimonio

de los grupos económicos mexicanos, 2005-2007

(En porcentajes)

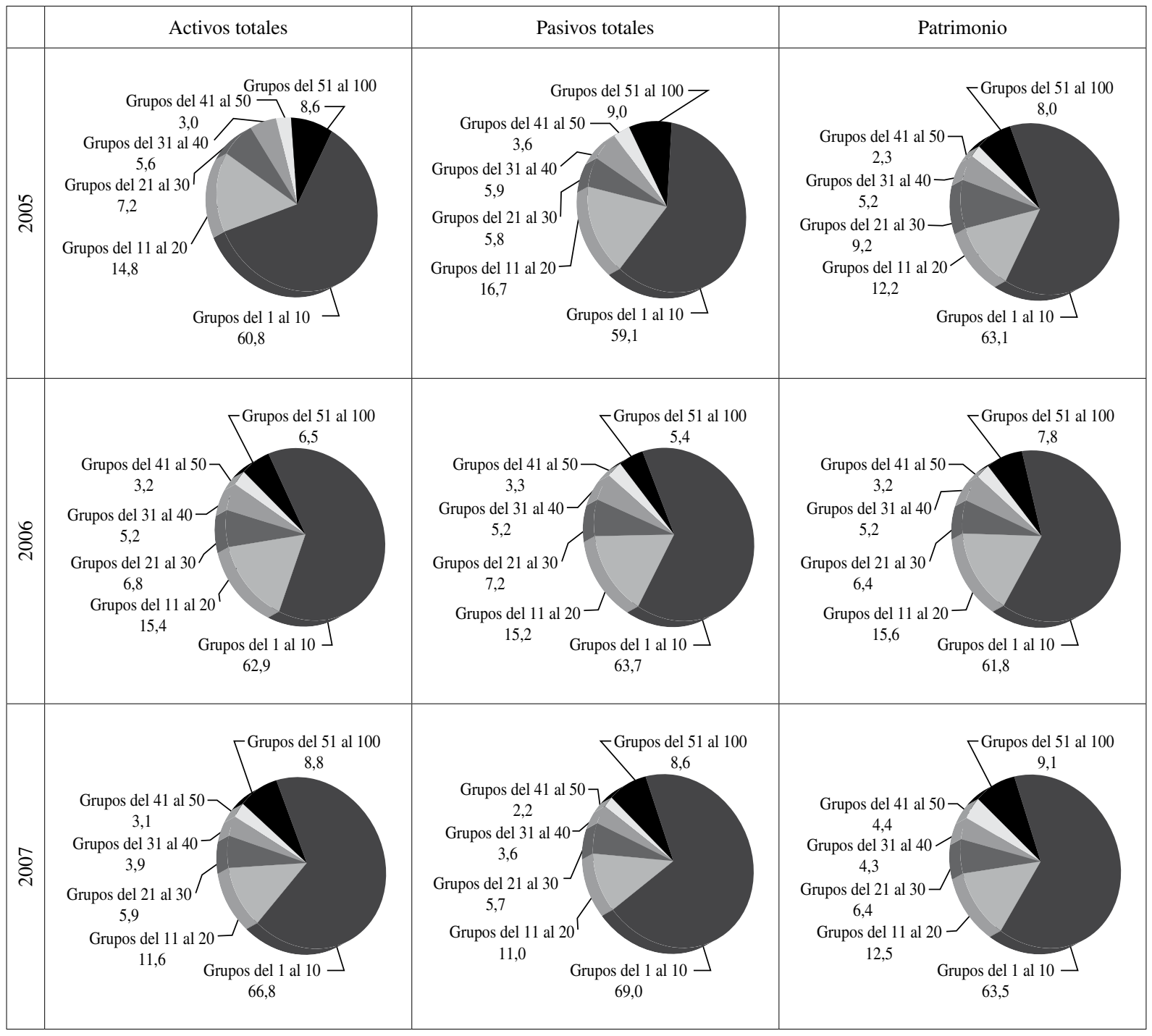

Fuente: elaboración propia sobre la base de datos obtenidos de la revista Expansión y del Instituto Nacional de Estadística, Geografía e Informática (INEGI).

como la suma de cuadrados de las participaciones de los activos totales de cada decil en el total de los 100 grupos. ${ }^{3}$ El valor máximo de IHH es 10.000 cuando

${ }^{3} \mathrm{IHH}=\sum_{i=1}^{n} S_{i}^{2}$, donde $S_{i}$ es la participación de cada grupo en el
total. un decil concentra el $100 \%$ del total de los activos (concentración máxima), desconcentrado $(\mathrm{IHH}<1.000)$, moderadamente concentrado $(1.000<\mathrm{IHH}<1.800) \mathrm{o}$ altamente concentrado ( $\mathrm{IHH}>1.800)$. Al respecto, con la información disponible es claro que la concentración de los activos es muy elevada y se incrementa en el tiempo, ya que el IHн de 2005 fue 4.506,4, en 2006 fue 4.672,5 y en 2007 ascendió a 5.208,7. 


\section{IV \\ Dinámica de crecimiento de los grupos económicos, 2004-2007}

En el cuadro 3 se consigna la información relativa a las principales partidas contables del total de los empresarios mexicanos para los años 2004 al 2007 en millones de pesos corrientes. Se consideran los activos totales, pasivos totales, patrimonio, ventas totales y utilidades netas. En dicho cuadro se incluye no solo la información para el total, sino la concerniente a los cinco primeros deciles (posiciones del 1 al 10, 11 al 20, 21 al 30, 31 al 40,41 al 50) y el subtotal de los valores para las empresas ubicadas en las posiciones del 51 al 100.
Un primer elemento que resalta es que entre 2004 y 2005 se reducen las ventas totales, los activos totales, los pasivos y el patrimonio de todos los empresarios. Entre esos años solo se incrementan las utilidades netas. Este fenómeno puede ser explicado mediante varias hipótesis. En primer lugar, puede obedecer a la presencia de ajustes en los criterios contables dispuestos por las autoridades gubernamentales, relativos a la consolidación de las empresas de un grupo económico. En segundo lugar, puede derivarse de la combinación

CUADRO 3

Valores de las principales partidas contables

de los grupos económicos mexicanos, 2004-2007

(En millones de pesos corrientes)

\begin{tabular}{|c|c|c|c|c|c|c|}
\hline Grupo & Año & Activos totales & Pasivos totales & Patrimonio total & Ventas & Utilidades netas \\
\hline \multirow[t]{4}{*}{ Grupos del 1 al 10} & 2004 & 2265072,0 & 1374430,0 & 890642,0 & 1314944,0 & 137250,0 \\
\hline & 2005 & 1722874,9 & 966419,7 & 756455,3 & 1141545,5 & 139267,8 \\
\hline & 2006 & 1615730,7 & 888420,5 & 727310,3 & 1302136,5 & 160293,4 \\
\hline & 2007 & 1934035,2 & 1192394,4 & 741640,7 & 1579250,8 & 214041,2 \\
\hline \multirow[t]{4}{*}{ Grupos del 11 al 20} & 2004 & 291554,0 & 143978,0 & 147576,0 & 284168,0 & 22857,0 \\
\hline & 2005 & 419231,0 & 272472,1 & 146758,8 & 283991,4 & 26053,7 \\
\hline & 2006 & 395449,6 & 211700,8 & 183748,8 & 423366,5 & 32995,3 \\
\hline & 2007 & 335344,7 & 189553,9 & 145790,9 & 375056,5 & 24341,3 \\
\hline \multirow[t]{4}{*}{ Grupos del 21 al 30} & 2004 & 167222,0 & 96864,0 & 70358,0 & 134669,0 & 4128,0 \\
\hline & 2005 & 205516,2 & 94855,3 & 110660,8 & 194277,1 & 10948,0 \\
\hline & 2006 & 175905,6 & 100453,0 & 75452,6 & 209954,2 & 11956,9 \\
\hline & 2007 & 172219,9 & 97955,0 & 74264,9 & 242440,5 & 13920,8 \\
\hline \multirow[t]{4}{*}{ Grupos del 31 al 40} & 2004 & 126921,0 & 63579,0 & 63342,0 & 103838,0 & 4481,0 \\
\hline & 2005 & 159153,1 & 96442,0 & 62711,0 & 144607,1 & 6471,5 \\
\hline & 2006 & 133482,0 & 72909,0 & 60573,1 & 102255,7 & 7164,3 \\
\hline & 2007 & 111558,2 & 61808,5 & 49749,7 & 141402,5 & 4417,6 \\
\hline \multirow[t]{4}{*}{ Grupos del 41 al 50} & 2004 & 70022,0 & 38544,0 & 31478,0 & 101312,0 & 3496,0 \\
\hline & 2005 & 85849,4 & 58681,8 & 27167,6 & 36942,6 & 4456,6 \\
\hline & 2006 & 82866,0 & 45510,7 & 37355,3 & 80883,5 & 4314,3 \\
\hline & 2007 & 88971,3 & 38078,4 & 50893,0 & 56676,4 & 8801,8 \\
\hline \multirow[t]{4}{*}{ Grupos del 51 al 100} & 2004 & 184840,0 & 114764,0 & 70076,0 & 129632,0 & 1591,0 \\
\hline & 2005 & 242684,5 & 146697,8 & 95986,7 & 137580,6 & 6647,1 \\
\hline & 2006 & 167056,0 & 75553,1 & 91502,8 & 163248,5 & 6995,0 \\
\hline & 2007 & 253811,9 & 147997,0 & 105814,9 & 166019,6 & 9283,2 \\
\hline \multirow[t]{4}{*}{ Total } & 2004 & 3105631,0 & 1832159,0 & 1273472,0 & 2068563,0 & 173803,0 \\
\hline & 2005 & 2835309,1 & 1635568,8 & 1199740,3 & 1938944,3 & 193844,7 \\
\hline & 2006 & 2570489,9 & 1394547,1 & 1175942,8 & 2281844,9 & 223719,2 \\
\hline & 2007 & 2895941,2 & 1727787,2 & 1168154,0 & 2560846,3 & 274805,9 \\
\hline
\end{tabular}

Fuente: elaboración propia sobre la base de datos obtenidos de la revista Expansión y del Instituto Nacional de Estadística, Geografía e Informática (INEGI). 
de los criterios contables mencionados anteriormente con la venta de activos o patrimonio por parte de algunos grupos nacionales al capital extranjero, aunque la información sobre la inversión extranjera directa para esos años no muestra un aumento significativo de los activos en manos de extranjeros.

Por otra parte, es importante consignar que las modificaciones suscitadas entre 2004 y 2005 en algunos de los 10 primeros grupos mexicanos (Slim, Larrea, Fernández, Garza Medina y Salinas Pliego) permitirían explicar la mayor parte de los montos observados para los 100 principales grupos empresariales del país. El mayor nivel de los activos totales, los pasivos y el patrimonio en 2004 respecto del 2005 se presenta también en los deciles de los grupos económicos de menor dimensión, a excepción de los ubicados en las posiciones del 41 al 50.

De 2005 a 2007 se produce un incremento en las ventas para el total de los 100 principales empresarios nacionales. Los activos totales nuevamente decrecen entre 2005 y 2006, para elevarse entre 2006 y 2007. En los pasivos totales se aprecia igual movimiento, contrayéndose nuevamente entre 2005 y 2006, para aumentar de 2006 a 2007. En el patrimonio, a pesar de que se engrosan las utilidades año con año, se observa una ligera tendencia decreciente a lo largo del tiempo.

En el cuadro 4 se presentan los flujos financieros anuales de los activos totales, los que deben explicarse por las variaciones en los pasivos totales y en el patrimonio para todos los 100 grandes empresarios mexicanos (y presentados por deciles). Solo se muestra la información para los períodos 2005-2006 y 2006-2007, ya que el período 2004-2005 involucra los elementos metodológicos comentados anteriormente. En virtud de que se trabaja con información de dos años en particular, no se considera necesario transformarla de millones de pesos corrientes a constantes.

Una primera anotación sobre la información disponible es que los mayores niveles de ventas no van acompañados de modificaciones en los activos totales: financieros, fijos o de intangibles. Estos niveles, relacionados con una demanda interna o externa más elevada, se derivan de una mayor rotación en los activos totales y de decisiones de inversión que se producen de manera discontinua en el tiempo. Con la reducida información disponible no es posible establecer otras hipótesis al respecto. De 2005 a 2006 hay una reducción de los activos totales que tiene su contrapartida en una mayor disminución de los pasivos totales respecto del patrimonio. Este fenómeno es general y puede deberse a razones de tipo contable $u$ otras.

Entre 2006 y 2007 se registra un importante crecimiento en los activos totales de los 100 empresarios analizados. Sin embargo, el comportamiento es disímil, ya que la mayor parte de este incremento se explicaría

CUADRO 4

Flujos financieros de los grupos mexicanos, 2005-2007

(En millones de pesos corrientes)

\begin{tabular}{lcccc}
\hline Grupos & Año & Variación de los activos totales & Variación de los pasivos totales & Variación del patrimonio \\
\hline \multirow{2}{*}{ Grupos del 1 al 10 } & $2005-2006$ & $-107144,2$ & $-77999,2$ & $-29145,0$ \\
& $2006-2007$ & 318304,5 & 303974,0 & 14330,5 \\
Grupos del 11al 20 & $2005-2006$ & $-23781,4$ & $-60771,3$ & 36989,9 \\
& $2006-2007$ & $-60104,9$ & $-22147,0$ & $-37957,9$ \\
Grupos del 21 al 30 & $2005-2006$ & $-29610,6$ & 5597,7 & $-35208,3$ \\
& $2006-2007$ & $-3685,7$ & $-2498,0$ & $-1187,7$ \\
Grupos del 31 al 40 & $2005-2006$ & $-25671,0$ & $-23533,1$ & $-138,0$ \\
& $2006-2007$ & $-21923,9$ & $-11100,5$ & 10187,4 \\
Grupos del 41 al 50 & $2005-2006$ & $-2983,4$ & $-13171,1$ & 13537,6 \\
& $2006-2007$ & 6105,3 & $-7432,4$ & $-4483,9$ \\
Grupos del 51 al 100 & $2005-2006$ & $-75628,6$ & $-71144,7$ & 14312,0 \\
Total 100 & $2006-2007$ & 86755,9 & 72443,9 & $-23797,5$ \\
& $2005-2006$ & $-264819,2$ & $-241021,7$ & $-7788,8$ \\
\hline
\end{tabular}

Fuente: elaboración propia sobre la base de datos obtenidos de la revista Expansión y del Instituto Nacional de Estadística, Geografía e Informática (INEGI).

a Empresas ordenadas según el ranking presentado en Expansión 2008 para el año 2007. 
por lo ocurrido en los 10 primeros grupos y en los menos importantes ubicados en las posiciones del 41 al 100. Resalta la reducción del valor de los activos y pasivos de los grupos ubicados en las posiciones del 11 al 40. La otra observación relevante, cuando se trabaja con el conjunto de las empresas, es que la variable de ajuste principal son los pasivos, mientras que el patrimonio tiene modificaciones menores.

En conjunto, los 100 mayores empresarios se financian sobre todo con capital de terceros (pasivos), mientras que las aportaciones de capital propio por medio del patrimonio son limitadas. La situación es obvia en el caso de los 10 primeros grupos empresariales cuya expansión de los activos totales se financia con pasivos y en menor proporción mediante el incremento del patrimonio. En cambio, la ampliación del patrimonio como mecanismo de financiamiento es más importante en los grupos vinculados a las posiciones del 41 al 100, en que destacan los empresarios ubicados en las posiciones del 41 al 50 cuya expansión de los activos totales se explica por un aumento del patrimonio y una reducción de los pasivos totales.

El financiamiento corporativo mediante los pasivos comprende el otorgado por el sistema bancario nacional, el sistema bancario internacional, la emisión de títulos de deuda colocados en los mercados locales e internacionales, entre los principales instrumentos financieros. Solo como referencia, es interesante constatar que entre 2006 y 2007 la expansión de los pasivos de los 100 primeros empresarios mexicanos fue equivalente al 76,2\% de la variación del crédito al sector privado del sistema financiero, de acuerdo con la información reportada por las Estadísticas financieras internacionales del Fondo Monetario Internacional (FMI, 2009).

En el cuadro 5 se muestran los resultados de dos regresiones de corte transversal mediante las cuales se pretende explicar el crecimiento de las ventas y de los activos totales, entre 2005 y 2007, de los grupos y empresarios reportados por Expansión. Se postula que el incremento de las ventas depende directamente de la rotación de los activos fijos y de la expansión de los activos totales, y estos últimos se explicarían más por el crecimiento de los pasivos que por el aporte de los accionistas (patrimonio). En los Anexos 1 y 2 se presenta la información básica para estas regresiones, la que se convierte a variaciones porcentuales para elevar el grado de exigencia en las regresiones.

CUADRO 5

Principales resultados de las regresiones de crecimiento en las ventas

\begin{tabular}{lcc}
\hline \multicolumn{1}{c}{ Variables } & \multicolumn{2}{c}{ Dependientes } \\
\cline { 2 - 3 } \multicolumn{1}{c}{ Independientes } & $\begin{array}{c}\text { Variación porcentual en ventas } \\
2005-2007\end{array}$ & $\begin{array}{c}\text { Variación porcentual en activos } \\
2005-2007\end{array}$ \\
\hline Constante & 24,3097 & $-3,0039$ \\
& $(-3,0426)$ & $(-1,8231)$ \\
Variación porcentual razón ventas/activos 2005-2007 & 0,2794 & \\
& $(10,1443)$ & 0,5563 \\
Variación porcentual activos totales 2005-2007 & 0,8348 & $(19,3295)$ \\
& $(4,4317)$ & 0,4618 \\
Variación porcentual pasivos totales 2005-2007 & & $(7,4837)$ \\
& & $-0,0002$ \\
Variación porcentual patrimonio 2005-2007 & & $(-0,1210)$ \\
Variación porcentual utilidades 2005-2007 & & 0,9092 \\
$\mathrm{R}^{2}$ & & 0,9050 \\
$\mathrm{R}^{2}$ ajustado & & 216,9358 \\
Prueba F & & 1,9499 \\
Test de Durbin-Watson & 0,6093 \\
\hline
\end{tabular}

Fuente: elaboración propia sobre la base de datos obtenidos de la revista Expansión y del Instituto Nacional de Estadística, Geografía e Informática (INEGI).

Nota: Debajo de los valores de los parámetros se muestra el valor de la prueba t. 
Al observar estos resultados, se concluye que mediante la primera regresión no se rechaza la hipótesis de que la variación porcentual en el crecimiento de las ventas depende positivamente de la variación porcentual de la razón ventas totales sobre activos totales y de la variación porcentual de los activos totales. En la segunda ecuación tampoco se rechaza que la variación porcentual de los activos totales depende en primer lugar de la variación porcentual de los pasivos y en segundo término del patrimonio. No hay vinculación directa con la variación porcentual de las utilidades netas del ejercicio.

Las bondades de ajuste de ambas ecuaciones son de más del $60 \%$ y el $90 \%$, respectivamente, que son valores altos para regresiones de corte transversal y en variaciones porcentuales. En los parámetros se refleja la lógica de lo postulado, pues estos tienen los signos correctos y son significativamente diferentes de cero de acuerdo con la prueba t. En conjunto, de acuerdo con la prueba F, son también diferentes de cero. En ambos casos se consideraron los datos para empresas que contaban con la información completa para el período 2005-2007: 69 en total. En ambas resgresiones no se detectaron problemas de autocorrelación, heterocedasticidad y multicolinealidad.

No se realizará un análisis detallado de las fuentes de crecimiento de los activos totales de los principales grupos empresariales o empresarios del país, ya que la información secundaria es todavía fragmentaria. Sin embargo, puede ser interesante anotar que de 2006 a 2007, de los 10 principales grupos nacionales, 4 aumentaron los niveles relativos de endeudamiento/activos (ZambranoCemex, Garza Medina-Alfa, Fernández González-Modelo y González Barrera-Maseca), 3 los mantuvieron (Slim, Bailléres-Bal y Salinas Pliego-Elektra) y 3 los redujeron (Larrea-Grupo México, Fernández Carbajal-Femsa y Servitje-Bimbo).

Por último, a diferencia de otros países, los grupos locales no cuentan en general con instituciones financieras bajo su control o que formen parte del grupo correspondiente. En 2007, de los 100 empresarios nacionales solo 12 contaban con instituciones financieras asociadas o que constituyen el núcleo central de sus actividades. En los 10 primeros grupos nacionales solo existen vinculaciones financieras directas en Slim-Grupo Financiero (GF) Inbursa y González Barrera-GF Banorte. En los grupos del 11 al 20 en solo 2: Salinas Pliego-Banco Elektra y Del Valle Ruiz-GF Ve por más. En las posiciones del 31 al 40 aparecen el GF Ixe y el GF Invex. En las posiciones del 51 al 100 figuran grupos propiamente financieros, pero de limitada envergadura nacional: Banco del Bajío, GF Banregio, GF Interacciones, GF Afirme, GF Mifel y GF Monex.

\section{V}

\section{Reflexiones finales}

La formación de los grupos económicos en México sigue la misma dinámica que lo ocurrido en la mayoría de los países de América Latina. Estos grupos surgieron principalmente con la industrialización temprana de finales del siglo XIX y comienzos del XX, durante el proceso de sustitución de importaciones entre los años cincuenta y setenta, y a partir de la reestructuración productiva neoliberal de los años ochenta. A lo largo del tiempo surgen nuevos grupos, otros mantienen su presencia, mientras algunos pierden importancia.

Las hipótesis propuestas para explicar el origen y funcionamiento de los grupos económicos son diversas. Una revisión extensiva de la literatura permite constatar que hay muchos elementos bajo discusión y que las evidencias concluyentes son limitadas. Coexisten principalmente los planteamientos en que se sostiene que los grupos surgen como resultado de las fallas o limitaciones en diversos mercados, pero también parecen tener asidero otras explicaciones sobre la vinculación de estos grupos con el Estado. Los grupos económicos mantienen una relación compleja con el Estado (dependiendo del gobierno), de apoyo mutuo, pero en determinadas circunstancias surgen los distanciamientos.

A partir de los estados financieros de cualquier grupo económico o empresa se pueden determinar las fuentes de expansión de los activos totales (financieros, fijos e intangibles), como contrapartida de las variaciones en los pasivos y el patrimonio. Las fuentes de expansión de los pasivos pueden encontrarse en el financiamiento bancario nacional e internacional, la emisión de deuda nacional e internacional y otros pasivos. En el caso del patrimonio, nos referimos a las utilidades reinvertidas y a las nuevas emisiones de acciones. Las proporciones entre pasivos y patrimonio se determinan de acuerdo con 
parámetros sectoriales, nacionales e internacionales, y según la propia evaluación del grupo financiero o empresario de que se trate.

Sobre la base de la información disponible, se aprecia que los grupos económicos mexicanos registran una creciente aportación al producto (PIB), lo que da cuenta de un proceso de concentración y centralización de ventas, activos totales, pasivos y patrimonio en manos de pocos grupos. La aportación de estos mismos grupos en términos del empleo y de los excedentes de explotación es de menor importancia. Sin embargo, la menor contribución al empleo obedece a mayores niveles de productividad respecto de las otras empresas que operan en la economía. No se ha realizado una evaluación de las empresas transnacionales y de los otros empresarios nacionales de menor dimensión.

Se observa un fenómeno de concentración en favor de los 10 primeros grupos económicos mexicanos y en desmedro de los otros grupos y empresarios registrados por Expansión. Los 10 primeros representan más del $60 \%$ de los activos totales, pasivos y patrimonio. Entre 2005 y 2007, llama la atención que esta participación es creciente en lo concerniente a los activos totales y pasivos, implicando un fenómeno de concentración. Al respecto, es interesante señalar que esta participación parece correlacionarse positivamente con los resultados, ya que el rendimiento sobre el patrimonio (roe) tiende también a crecer con el tiempo en el período bajo análisis.

Es claro que el crecimiento de las ventas de los grupos económicos se explica por incrementos en la rotación de los activos fijos (ventas totales/activos totales) y en los activos totales. Lo anterior resulta del análisis directo de la información financiera y de las regresiones de corte transversal. Asimismo, la inversión que realizan los diferentes grupos económicos no se produce de manera continua en el tiempo, sino que con saltos discretos (esporádicamente), aprovechando ventajas particulares que pueden vincularse al tipo de cambio real, a tasas de interés y a aranceles, entre otras.

Pese a que la mayor parte de los grupos económicos mexicanos no guardan tan estrechas relaciones con las empresas del sector financiero, como antes era característico, en el período bajo análisis de 2005 a 2007, la principal fuente de financiamiento de la expansión de los activos totales corresponde al crecimiento del capital de terceros (pasivos) y en menor proporción al incremento del capital propio (patrimonio). Los principales grupos económicos, en particular los 10 primeros, aprovechan su posición para obtener más capital de terceros mediante un mayor nivel de apalancamiento respecto del promedio de los grupos económicos y empresarios nacionales.

Entre los 15 grupos económicos de mayor dimensión destacan 3: Slim, González Barrera y Salinas Pliego, que han mostrado un gran dinamismo a partir de los años ochenta y cuentan con instituciones financieras propias. Por otra parte, los grupos económicos de menor dimensión ubicados en las posiciones del 41 al 100 requieren recurrir a capital propio más que a capital de terceros para financiar la expansión de los activos totales. Los ubicados en las posiciones del 41 al 50 autofinanciaron la expansión de sus activos totales en el período bajo análisis.

En la menor rotación de las ventas de los 10 principales grupos económicos nacionales se aprecia una constatación comentada por otros autores, cual es que como parte de sus estrategias defensivas estas empresas tienden a "sobreinvertir", generando barreras a la entrada de competidores. La menor rotación denotaría niveles inferiores de productividad con respecto al uso de los activos, pero estas empresas están dispuestas a asumir el costo de oportunidad por su subutilización a fin de garantizar los espacios de crecimiento para sus grupos. Asimismo, no hay que olvidar que probablemente estos grupos de mayor dimensión enfrentan costos financieros más reducidos que el resto de los grupos y empresarios de menor envergadura.

Por último, una constatación importante con relación a los grupos económicos mexicanos es que las redes empresariales pueden ser grandes y más o menos complejas, y contar con estrategias ofensivas y defensivas, pero la estructura de capital continúa siendo muy tradicional, donde son claros la presencia y el control de un grupo familiar en particular. Del mismo modo, llama la atención que ninguno de los grandes grupos mexicanos ocupa un lugar significativo en ramas de alta tecnología (Salas-Porras, 2006a, p. 6). 
ANEXO 1

Estadísticas básicas para las regresiones econométricas, 2005-2007

(En millones de pesos corrientes)

\begin{tabular}{|c|c|c|c|c|c|c|}
\hline \multirow{2}{*}{ Grupos económicos / Empresarios } & \multicolumn{2}{|c|}{ Ventas } & \multicolumn{2}{|c|}{ Activos totales } & \multicolumn{2}{|c|}{ Pasivos totales } \\
\hline & 2005 & 2007 & 2005 & 2007 & 2005 & 2007 \\
\hline
\end{tabular}

Grupo Carso, América Móvil, Imbursa (Carlos Slim Helú e hijos) Cemex (Lorenzo Zambrano Treviño)

Grupo México (Germán Larrea Mota Velasco)

Fomento Económico Mexicano (José Antonio Fernández Carbajal) Grupo Alfa (Dionisio Garza Medina)

Grupo Modelo (Carlos Fernández González)

Gruma y Banorte (Roberto González Barrera)

Grupo Bimbo (Daniel Servitje Montull)

Grupo Bal (Alberto Bailléres González)

Grupo Salinas Elektra (Ricardo Salinas Pliego)

Organización Soriana (Ricardo Martín Bringas)

Grupo Televisa (Emilio Azcárraga Jean)

Mexichem (Antonio del Valle Ruiz)

Grupo Coppel (Enrique Coppel Luken)

Empresas ICA (Bernardo Quintana Isaac)

Comercial Mexicana (Guillermo González Nova y Carlos González Zabalegui)

Liverpool (Max David Michel y Max Michel Souberville)

Axtel y Avantel (Tomás Milmo Santos)

Grupo Xignux (Eugenio Garza Herrera)

Homex (Eustaquio Tomás de Nicolás Gutiérrez)

Bachoco (Francisco Robinson Bours-Castelo)

Grupo Acerero del Norte (Xavier Autrey Maza y Alonso Ancira Elizondo)

Casas Geo (Luis Orvañanos Lascurain)

Arca (Manuel Barragán Morales y Miguel Fernández Iturriza)

Kimberly-Clark (Claudio X. González Laporte y Pablo González Guajardo)

Fragua (Javier Arroyo Chávez)

Grupo Posadas (Gastón Azcárraga Andrade)

Grupo Simec (Rufino Vigil González)

Urbi (Cuauhtémoc Pérez Román Urbi)

Grupo Kuo (Fernando Senderos Mestre)

Corporación Durango (Miguel Rincón Arredondo)

Quálitas Compañía de Seguros (Joaquín Brockman Lozano)

Alsea (Cosme Alberto Torrado Martínez)

Grupo Lamosa (Federico Toussaint Elosúa)

Grupo Continental (Cynthia Helena Grossman)

Interacciones (Carlos Hank Rhon)

Grupo Embotelladoras Unidas (Juan Gallardo Thurlow)

Consorcio ARA (Germán Ahumada Russek)

Minera Autlán (José Antonio Rivero Larrea)

Grupo Cementos de Chihuahua (Federico Terrazas Torres)

Grupo Aeroportuario del Sureste (Fernando Chico Pardo)

Ixe Grupo Financiero (Enrique Luis Castillo Sánchez Mejorada)

Grupo Gigante (Ángel Losada Moreno)

Promotora Ambiental (Alberto Garza Santos)

Verzatec (Eugenio Clariond Reyes)

Promotora y Operación de Infraestructura (David Peñalosa Sandoval)

Coorporación Interamericana de Entretenimiento (Alejandro Soberón Kuri) SANLUIS Rassini (Antonio Madero Bracho)

Grupo Transportación Marítima Mexicana (José Francisco Serrano Segovia)

Grupo Martí (Alfredo Harp Helú)

Internacional de Cerámica (Óscar Almeida Chabre)

Corporacion Mexicana de Restaurantes (Joaquín Vargas Guajardo)

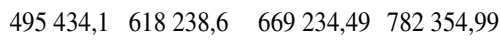
$162708,6 \quad 236669,0 \quad 293138,56 \quad 370126,53$

$\begin{array}{llll}58101,8 & 80950,2 & 99512,20 & 81865,12\end{array}$

$\begin{array}{llll}105581,7 & 147556,1 & 127642,28 & 99505,10\end{array}$

$\begin{array}{lllll}69334,8 & 106832,7 & 61966,70 & 74323,26\end{array}$

$\begin{array}{lllll}49550,5 & 72894,6 & 80311,38 & 71325,47\end{array}$

$\begin{array}{lllll}63856,4 & 97308,6 & 240468,45 & 224 & 355,33\end{array}$

$\begin{array}{llll}56102,2 & 72293,6 & 36270,51 & 36797,82\end{array}$

$\begin{array}{lllll}57278,4 & 85127,4 & 76248,60 & 104607,88\end{array}$

$\begin{array}{lllll}47075,9 & 61380,0 & 70101,08 & 88773,67\end{array}$

$\begin{array}{llll}48394,4 & 65190,7 & 35798,32 & 54005,17\end{array}$

$\begin{array}{llll}32481,0 & 41561,5 & 78532,05 & 85121,11\end{array}$

$\begin{array}{rrrr}8677,4 & 24236,9 & 91030,97 & 16104,43\end{array}$

$19607,5 \quad 34752,7$

$18404,9 \quad 22447,8$

$40308,9 \quad 50409,2$

$32055,2 \quad 42976,3$

$4966,8 \quad 12190,6$

$21983,1 \quad 39142,7$

$8571,4 \quad 16166,1$

$14437,4 \quad 18208,8$

$22718,0 \quad 27379,0$

$10091,3 \quad 14975,6$

$14647,0 \quad 18572,7$

$21983,1 \quad 21480,2$

$9832,2 \quad 14575,2$

$5126,8 \quad 25948,9$

$16219,0 \quad 27640,1$

$8194,3 \quad 12779,4$

$24577,4 \quad 22722,8$

$8150,4 \quad 15430,0$

$4450,3 \quad 8593,4$

$4318,3 \quad 7047,3$

$3782,0 \quad 6817,3$

$10623,8 \quad 12283,3$

$1773,3 \quad 11055,1$

$6304,3 \quad 7713,6$

$6772,7 \quad 9257,3$

$2097,9 \quad 2461,7$

$4722,9 \quad 8453,2$

$2063,8 \quad 2785,9$

$2261,7 \quad 6460,0$

$32524,6 \quad 27115,3$

$2096,5 \quad 3004,5$

$39182,1 \quad 7148,4$

$2645,8 \quad 2715,5$

$8658,9 \quad 10187,9$

$6841,0 \quad 7999,7$

$3260,5 \quad 3306,8$

$2322,7 \quad 3282,0$

$4462,0 \quad 5503,4$

$1137,4 \quad 1643,7$
$18733,54 \quad 33933,85$

$31241,39 \quad 18372,94$

$30453,33 \quad 34886,57$

$36910,44 \quad 42109,51$

$10872,34 \quad 16789,51$

$15352,04 \quad 12737,86$

$14143,73 \quad 17598,52$

$15286,38 \quad 17068,65$

$40265,28 \quad 36682,58$

$13495,70 \quad 14883,51$

$14178,57 \quad 15602,39$

$25247,33 \quad 22765,19$

$3811,36 \quad 4594,67$

$11865,63 \quad 17941,06$

$19314,24 \quad 19489,57$

$12376,05 \quad 16218,86$

$24460,23 \quad 20343,34$

$14400,00 \quad 14653,13$

$4955,84 \quad 11031,07$

$3039,07 \quad 4369,28$

$5494,06 \quad 11423,86$

$8900,13 \quad 9394,08$

$9412,80 \quad 3098,05$

$4664,87 \quad 3841,39$

$9685,22 \quad 11543,01$

$2667,11 \quad 2917,99$

$11159,42 \quad 15641,39$

$14114,58 \quad 15967,29$

$22641,67 \quad 15410,96$

$28514,10 \quad 27583,94$

$2477,71 \quad 2843,04$

$35469,47 \quad 33221,82$

$6450,18 \quad 10620,09$

$15672,84 \quad 9786,37$

$8190,48 \quad 3200,63$

$8631,50 \quad 12156,15$

$2969,70 \quad 3442,50$

$4465,51 \quad 4629,50$

$1092,86 \quad 1366,47$
371 796,94 488971,87

$183211,60231329,08$

$41463,41 \quad 29237,54$

$60782,04 \quad 45229,59$

$29507,95 \quad 41290,70$

$12168,39 \quad 12736,69$

$200390,37 \quad 186962,78$

$16486,60 \quad 13628,82$

$54463,29 \quad 74719,92$

$58417,57 \quad 68287,44$

$14915,97 \quad 30002,87$

$35696,39 \quad 50071,24$

$56894,35 \quad 11503,16$

$10407,52 \quad 22622,57$

$18377,29 \quad 8749,02$

$13842,42 \quad 15857,53$

$17576,40 \quad 19140,69$

$3749,08 \quad 9327,51$

$9030,61 \quad 7076,59$

$8319,84 \quad 10352,07$

$2779,34 \quad 3555,97$

$28760,92 \quad 24455,05$

$8434,81 \quad 8268,62$

$3832,05 \quad 4216,86$

$13288,07 \quad 13391,29$

$1732,44 \quad 1997,68$

$6592,01 \quad 13800,81$

$6230,40 \quad 4753,55$

$5625,48 \quad 7723,27$

$11647,73 \quad 11301,86$

$9600,00 \quad 9768,75$

$4129,87 \quad 10028,25$

$1266,28 \quad 1820,53$

$2616,22 \quad 8159,90$

$1934,81 \quad 1957,10$

$7844,00 \quad 1032,68$

$2028,21 \quad 1670,17$

$2934,92 \quad 4439,62$

$919,69 \quad 1080,74$

$3985,51 \quad 7109,72$

$1037,84 \quad 2073,67$

$20583,33 \quad 12842,47$

$12397,44 \quad 11493,31$

$952,96 \quad 1292,29$

$15421,51 \quad 10381,82$

$5863,80 \quad 9654,63$

$9795,52 \quad 6116,48$

$5119,05 \quad 2133,75$

$7192,91 \quad 10130,12$

$1414,14 \quad 1912,50$

$2480,84 \quad 2571,94$

$242,86 \quad 440,80$ 
(Continuación anexo 1)

\begin{tabular}{|c|c|c|c|c|c|c|}
\hline \multirow{2}{*}{ Grupos económicos / Empresarios } & \multicolumn{2}{|c|}{ Ventas } & \multicolumn{2}{|c|}{ Activos totales } & \multicolumn{2}{|c|}{ Pasivos totales } \\
\hline & 2005 & 2007 & 2005 & 2007 & 2005 & 2007 \\
\hline Mifiel Grupo Financiero (Daniel Becker Feldman) & 1248,7 & 2597,1 & 4877,78 & 14532,45 & 4064,81 & 13211,31 \\
\hline Cydsa (Tomás González Sada) & 5355,4 & 6186,4 & 8071,56 & 6833,16 & 3668,89 & 2628,14 \\
\hline Invex Grupo Financiero (Juan Guichard Michel) & 889,7 & 3976,9 & 9137,36 & 11682,19 & 7028,74 & 8986,30 \\
\hline Grupo Mexicano de Desarrollo (Jorge Ballesteros Franco) & 1080,1 & 1517,9 & 3866,10 & 3734,13 & 1431,89 & 1383,01 \\
\hline Grupo Collado (Guillermo Vogel Hinojosa) & 4954,2 & 5883,2 & 3371,43 & 4330,19 & 2107,14 & 3092,99 \\
\hline Médica Sur (Misael Uribe Esquivel y Manuel Martínez López) & 1069,4 & 1397,2 & 1566,65 & 1742,52 & 540,23 & 528,04 \\
\hline Monex Grupo Financiero (Héctor Lagos Cué y Héctor Lagos Dondé) & 1335,0 & 2232,0 & 2399,51 & 1975,28 & 1090,69 & 1097,38 \\
\hline Grupo Industrial Saltillo (Ernesto López de Nigris y Juan Carlos López Villarreal) & 9518,2 & 8823,7 & 12366,67 & 10480,18 & 6183,33 & 4556,60 \\
\hline Grupo Famsa (Humberto Garza González) & 10611,3 & 14181,2 & 10968,49 & 14029,05 & 7312,33 & 8252,38 \\
\hline Grupo Casa Saba (Isaac Saba Raffoul) & 21829,3 & 25126,5 & 10008,28 & 11157,17 & 5267,52 & 5872,20 \\
\hline Grupo Bafar (Óscar Eugenio Baeza Farés) & 3407,6 & 4318,7 & 2741,74 & 3330,13 & 1096,70 & 1189,33 \\
\hline Grupo la Moderna (Eduardo Monroy Cárdenas) & 3676,1 & 4651,8 & 4216,38 & 4035,01 & 795,54 & 733,64 \\
\hline Grupo Ruba (Enrique Terrazas Torres) & 2557,0 & 4567,6 & 2511,22 & 3587,37 & 1141,47 & 1559,72 \\
\hline Copamex (Juan Bosco Maldonado Quiroga) & 4724,7 & 5562,5 & 6800,00 & 7287,80 & 3400,00 & 3643,90 \\
\hline Grupo Minsa (Raymundo, Armando, Alfonso y Guillermo Gómez Flores) & 2214,2 & 3033,1 & 2085,12 & 2604,64 & 347,52 & 685,43 \\
\hline Grupo Accel (Eloy Vallina Lagüera) & 1537,3 & 1726,1 & 1713,13 & 1628,85 & 535,35 & 542,95 \\
\hline
\end{tabular}

Fuente: elaboración propia sobre la base de datos obtenidos de la revista Expansión y del Instituto Nacional de Estadística, Geografía e Informática (INEGI).

ANEXO 2

Estadísticas básicas para las regresiones econométricas, 2005-2007 (En millones de pesos corrientes)

\begin{tabular}{|c|c|c|c|c|c|c|}
\hline \multirow{2}{*}{ Grupos económicos / Empresarios } & \multicolumn{2}{|c|}{ Patrimonio } & \multicolumn{2}{|c|}{$\begin{array}{l}\text { Utilidad } \\
\text { neta }\end{array}$} & \multicolumn{2}{|c|}{$\begin{array}{c}\text { Razón } \\
\text { Ventas/Activos }\end{array}$} \\
\hline & 2005 & 2007 & 2005 & 2007 & 2005 & 2007 \\
\hline Grupo Carso América Móvil Imbursa (Carlos Slim Helú e hijos) & 297437,55 & 293383,12 & 70492,7 & 122370,1 & 0,74 & 0,79 \\
\hline Cemex (Lorenzo Zambrano Treviño) & 109926,96 & 138797,45 & 22425,1 & 26107,8 & 0,56 & 0,64 \\
\hline Grupo México (Germán Larrea Mota Velasco) & 58048,78 & 52627,58 & 7140,0 & 18356,5 & 0,58 & 0,99 \\
\hline Fomento Económico Mexicano (José Antonio Fernández Carbajal) & 66860,24 & 54275,51 & 5549,4 & 8510,4 & 0,83 & 1,48 \\
\hline Grupo Alfa (Dionisio Garza Medina) & 32458,75 & 33032,56 & 7790,1 & 3551,0 & 1,12 & 1,44 \\
\hline Grupo Modelo (Carlos Fernández González) & 68142,99 & 58588,78 & 7291,3 & 9503,1 & 0,62 & 1,02 \\
\hline Gruma y Banorte (Roberto González Barrera) & 40078,07 & 37392,56 & 7494,6 & 9744,5 & 0,27 & 0,43 \\
\hline Grupo Bimbo (Daniel Servitje Montull) & 19783,92 & 23169,00 & 2829,1 & 3811,3 & 1,55 & 1,96 \\
\hline Grupo Bal (Alberto Bailléres González) & 21785,31 & 29887,97 & 3115,3 & 5762,4 & 0,75 & 0,81 \\
\hline Grupo Salinas Elektra (Ricardo Salinas Pliego) & 11683,51 & 20486,23 & 5386,1 & 6324,1 & 0,67 & 0,69 \\
\hline Organización Soriana (Ricardo Martín Bringas) & 20882,35 & 24002,30 & 2130,0 & 3134,7 & 1,35 & 1,21 \\
\hline Grupo Televisa (Emilio Azcárraga Jean) & 42835,66 & 35049,87 & 6125,5 & 8082,5 & 0,41 & 0,49 \\
\hline Mexichem (Antonio del Valle Ruiz) & 34136,61 & 4601,27 & 6247,0 & 2108,3 & 0,10 & 1,50 \\
\hline Grupo Coppel (Enrique Coppel Luken) & 8326,02 & 11311,28 & 1024,1 & 2616,3 & 1,05 & 1,02 \\
\hline Empresas ICA (Bernardo Quintana Isaac) & 12864,10 & 9623,92 & 501,7 & $-893,1$ & 0,59 & 1,22 \\
\hline Comercial Mexicana (Guillermo González Nova y Carlos González Zabalegui) & 16610,91 & 19029,04 & 1827,2 & 2555,6 & 1,32 & 1,44 \\
\hline Liverpool (Max David Michel y Max Michel Souberville) & 19334,04 & 22968,82 & 2726,1 & 3831,2 & 0,87 & 1,02 \\
\hline Axtel y Avantel (Tomás Milmo Santos) & 7123,26 & 7462,01 & 306,3 & 491,0 & 0,46 & 0,73 \\
\hline Grupo Xignux (Eugenio Garza Herrera) & 6321,43 & 5661,27 & 531,0 & 1049,6 & 1,43 & 3,07 \\
\hline Homex (Eustaquio Tomás de Nicolás Gutiérrez) & 5823,89 & 7246,45 & 1048,3 & 2193,5 & 0,61 & 0,92 \\
\hline Bachoco (Francisco Robinson Bours-Castelo) & 12507,04 & 13512,68 & 1776,0 & 1278,3 & 0,94 & 1,07 \\
\hline Grupo Acerero del Norte (Xavier Autrey Maza y Alonso Ancira Elizondo) & 11504,37 & 12227,53 & 2634,5 & 1972,3 & 0,56 & 0,75 \\
\hline Casas Geo (Luis Orvañanos Lascurain) & 5060,89 & 6614,89 & 1138,7 & 1448,0 & 0,75 & 1,01 \\
\hline
\end{tabular}


(Continuación anexo 2)

\begin{tabular}{|c|c|c|c|c|c|c|}
\hline \multirow{2}{*}{ Grupos económicos / Empresarios } & \multicolumn{2}{|c|}{ Patrimonio } & \multicolumn{2}{|c|}{$\begin{array}{l}\text { Utilidad } \\
\text { neta }\end{array}$} & \multicolumn{2}{|c|}{$\begin{array}{c}\text { Razón } \\
\text { Ventas/Activos }\end{array}$} \\
\hline & 2005 & 2007 & 2005 & 2007 & 2005 & 2007 \\
\hline Arca (Manuel Barragán Morales y Miguel Fernández Iturriza) & 10346,52 & 11385,53 & 1934,8 & 2501,4 & 1,03 & 1,19 \\
\hline Kimberly-Clark (Claudio X, González Laporte y Pablo González Guajardo) & 11959,26 & 9373,90 & 2906,1 & 3728,0 & 0,87 & 0,94 \\
\hline Fragua (Javier Arroyo Chávez) & 2078,92 & 2596,99 & 463,6 & 672,1 & 2,58 & 3,17 \\
\hline Grupo Posadas (Gastón Azcárraga Andrade) & 5273,61 & 4140,24 & 379,7 & 135,8 & 0,43 & 1,45 \\
\hline Grupo Simec (Rufino Vigil González) & 13083,84 & 14736,02 & 1295,3 & 1791,9 & 0,84 & 1,42 \\
\hline Urbi (Cuauhtémoc Pérez Román Urbi) & 6750,57 & 8495,60 & 1174,6 & 1832,5 & 0,66 & 0,79 \\
\hline Grupo Kuo (Fernando Senderos Mestre) & 12812,50 & 9041,48 & 307,5 & $-828,2$ & 1,00 & 1,12 \\
\hline Corporación Durango (Miguel Rincón Arredondo) & 4800,00 & 4884,38 & 52,8 & $-312,6$ & 0,57 & 1,05 \\
\hline Quálitas Compañía de Seguros (Joaquín Brockman Lozano) & 825,97 & 1002,82 & $-63,6$ & 35,5 & 0,90 & 0,78 \\
\hline Alsea (Cosme Alberto Torrado Martínez) & 1772,79 & 2548,75 & 260,6 & 478,4 & 1,42 & 1,61 \\
\hline Grupo Lamosa (Federico Toussaint Elosúa) & 2877,84 & 3263,96 & 506,5 & 900,2 & 0,69 & 0,60 \\
\hline Grupo Continental (Cynthia Helena Grossman) & 6965,32 & 7436,98 & 1205,0 & 1604,9 & 1,19 & 1,31 \\
\hline Interacciones (Carlos Hank Rhon) & 1568,80 & 2065,37 & 196,1 & 603,5 & 0,19 & 3,57 \\
\hline Grupo Embotelladoras Unidas (Juan Gallardo Thurlow) & 2636,67 & 2171,22 & 158,2 & 294,2 & 1,35 & 2,01 \\
\hline Consorcio ARA (Germán Ahumada Russek) & 6750,31 & 7103,39 & 1100,3 & 1339,7 & 0,70 & 0,80 \\
\hline Minera Autlán (José Antonio Rivero Larrea) & 1747,41 & 1837,25 & 202,7 & 296,9 & 0,79 & 0,84 \\
\hline Grupo Cementos de Chihuahua (Federico Terrazas Torres) & 7173,91 & 8531,66 & 990,0 & 1670,5 & 0,42 & 0,54 \\
\hline Grupo Aeroportuario del Sureste (Fernando Chico Pardo) & 13076,74 & 13893,62 & 562,3 & 522,4 & 0,15 & 0,17 \\
\hline Ixe Grupo Financiero (Enrique Luis Castillo Sánchez Mejorada) & 2058,33 & 2568,49 & 123,5 & $-75,0$ & 0,10 & 0,42 \\
\hline Grupo Gigante (Ángel Losada Moreno) & 16116,67 & 16090,63 & $-96,7$ & 4722,6 & 1,14 & 0,98 \\
\hline Promotora Ambiental (Alberto Garza Santos) & 1524,74 & 1550,75 & 147,9 & 93,2 & 0,85 & 1,06 \\
\hline Verzatec (Eugenio Clariond Reyes) & 20047,96 & 22840,00 & 1964,7 & 285,5 & 1,10 & 0,22 \\
\hline Promotora y Operación de Infraestructura (David Peñalosa Sandoval) & 586,38 & 965,46 & 163,6 & 547,9 & 0,41 & 0,26 \\
\hline Coorporación Interamericana de Entretenimiento (Alejandro Soberón Kuri) & 5877,31 & 3669,89 & $-1269,5$ & $-569,2$ & 0,55 & 1,04 \\
\hline SANLUIS Rassini (Antonio Madero Bracho) & 3071,43 & 1066,88 & $-21,5$ & $-982,7$ & 0,84 & 2,50 \\
\hline Grupo Transportación Marítima Mexicana (José Francisco Serrano Segovia) & 1438,58 & 2026,02 & 1827,0 & $-731,8$ & 0,38 & 0,27 \\
\hline Grupo Martí (Alfredo Harp Helú) & 1555,56 & 1530,00 & 112,0 & 76,5 & 0,78 & 0,95 \\
\hline Internacional de Cerámica (Óscar Almeida Chabre) & 1984,67 & 2057,55 & 271,9 & 28,6 & 1,00 & 1,19 \\
\hline Corporacion Mexicana de Restaurantes (Joaquín Vargas Guajardo) & 850,00 & 925,68 & 47,6 & 82,2 & 1,04 & 1,20 \\
\hline Mifiel Grupo Financiero (Daniel Becker Feldman) & 812,96 & 1321,13 & 87,8 & 79,4 & 0,26 & 0,18 \\
\hline Cydsa (Tomás González Sada) & 4402,67 & 4205,02 & $-330,2$ & 100,5 & 0,66 & 0,91 \\
\hline Invex Grupo Financiero (Juan Guichard Michel) & 2108,62 & 2695,89 & 122,3 & 196,8 & 0,10 & 0,34 \\
\hline Grupo Mexicano de Desarrollo (Jorge Ballesteros Franco) & 2434,21 & 2351,12 & 185,0 & 136,6 & 0,28 & 0,41 \\
\hline Grupo Collado (Guillermo Vogel Hinojosa) & 1264,29 & 1237,20 & 53,1 & $-45,9$ & 1,47 & 1,36 \\
\hline Médica Sur (Misael Uribe Esquivel y Manuel Martínez López) & 1026,43 & 1214,49 & 143,7 & 216,3 & 0,68 & 0,80 \\
\hline Monex Grupo Financiero (Héctor Lagos Cué y Héctor Lagos Dondé) & 1308,82 & 877,90 & 267,0 & 370,3 & 0,56 & 1,13 \\
\hline Grupo Industrial Saltillo (Ernesto López de Nigris y Juan Carlos López Villarreal) & 6183,33 & 5923,58 & 74,2 & 364,3 & 0,77 & 0,84 \\
\hline Grupo Famsa (Humberto Garza González) & 3656,16 & 5776,67 & 266,9 & 519,9 & 0,97 & 1,01 \\
\hline Grupo Casa Saba (Isaac Saba Raffoul) & 4740,76 & 5284,98 & 744,3 & 921,7 & 2,18 & 2,25 \\
\hline Grupo Bafar (Óscar Eugenio Baeza Farés) & 1645,05 & 2140,80 & 365,2 & 193,1 & 1,24 & 1,30 \\
\hline Sare (Dionisio Sánchez Carbajal) & 3031,43 & 2810,16 & 318,3 & 487,0 & 0,68 & 0,91 \\
\hline Grupo la Moderna (Eduardo Monroy Cárdenas) & 3420,83 & 3301,37 & 328,4 & 361,5 & 0,87 & 1,15 \\
\hline Grupo Ruba (Enrique Terrazas Torres) & 1369,76 & 2027,64 & 339,7 & 579,5 & 1,02 & 1,27 \\
\hline Copamex (Juan Bosco Maldonado Quiroga) & 3400,00 & 3643,90 & 17,0 & 74,7 & 0,69 & 0,76 \\
\hline Grupo Minsa (Raymundo Armando Alfonso y Guillermo Gómez Flores) & 1737,60 & 1919,21 & 420,5 & 125,9 & 1,06 & 1,16 \\
\hline Grupo Accel (Eloy Vallina Lagüera) & 1177,78 & 1085,90 & 21,2 & 49,3 & 0,90 & 1,06 \\
\hline
\end{tabular}

Fuente: elaboración propia sobre la base de datos obtenidos de la revista Expansión y del Instituto Nacional de Estadística, Geografía e Informática (INEGI). 
Basave, J. y M. Hernández (coords.) (2007), Los estudios de empresarios y empresa: una perspectiva internacional, México, D.F., Instituto de Investigaciones Económicas, Universidad Nacional Autónoma de México.

Batchikov, S. e I. Petrov (1997), "The formation of financial-industrial groups and the estate", Russian and East European Finance and Trade, vol. 33, $\mathrm{N}^{\circ} 1$, Armonk, M.E. Sharpe, Inc., enerofebrero.

Calderón, Á. (2006), "El modelo de expansión de las grandes cadenas minoristas chilenas", Revista de la CEPAL, No 90 (LC/G.2323-P), Santiago de Chile, Comisión Económica para América Latina y el Caribe (CEPAL), diciembre.

Castañeda, G. (2004), "Los grupos económicos como estabilizadores financieros de la economía mexicana 1996-2000”, Problemas del desarrollo, vol. 35, No 136, México, D.F., Instituto de Investigaciones Económicas, Universidad Nacional Autónoma de México, enero-marzo.

Cerutti, M., I. Ortega y Lylia Palacios (2000), "Empresarios y empresas en el norte de México: Monterrey, del Estado oligárquico a la globalización", European Review of Latin American and Caribbean Studies, $\mathrm{N}^{\circ}$ 69, Amsterdam, Centro de Estudios y Documentación Latinoamericanos (CEDLA), octubre.

Chavarín, R. (2006), "La arquitectura organizacional y gobierno corporativo de los grupos económicos en México", Ciencia Ergo Sum, vol. 13, № 2, Toluca, Universidad Autónoma del Estado de México (UAMEX), julio-octubre.

Correa. E. (2000), "Conglomerados y reforma financiera", Comercio exterior, vol. 49, $\mathrm{N}^{\circ}$ 6, México, D.F., Bancomext, junio.

Expansión, S.A. de C.V. (2008), "Los 100 empresarios más importantes de México”, Expansión, México, D.F., Grupo Editorial Expansión, 28 de abril-11 de mayo.

(2007), "Los 100 empresarios más importantes de México", Expansión, México, D.F., Grupo Editorial Expansión, 30 de abril-15 de mayo.

(2006), "Los 100 empresarios más importantes de México", Expansión, México, D.F., Grupo Editorial Expansión, 31 de mayo-14 de junio.

(2005), "Los 100 empresarios más importantes de México", Expansión, México, D.F., Grupo Editorial Expansión, 4-18 de mayo.

Fernández, A. (2000), “América Latina: el debate sobre los nuevos grupos económicos y conglomerados industriales después de la reestructuración neoliberal", European Review of Latin American and Caribbean Studies, $\mathrm{N}^{\circ}$ 69, Amsterdam, Centro de Estudios y Documentación Latinoamericanos (CEDLA), octubre.

FMI (Fondo Monetario Internacional) (2009), Estadísticas financieras internacionales, Washington, D.C.

Garrido, C. (2002), "Industrialización y grandes empresas en el desarrollo estabilizador, 1958-1970", Análisis económico, vol. 17, $\mathrm{N}^{\circ} 35$, Azcapotzalco, D.F., Universidad Autónoma Metropolitana (UAM)

(2001), "Estrategias empresariales ante el cambio estructural en México", Comercio exterior, vol. 67, № 12, México, D.F., Bancomext, diciembre.

(1997), "Las grandes empresas privadas nacionales mexicanas", Nueva sociedad, № 151, Caracas, Fundación Friedrich Ebert, septiembre-octubre.

Garrido, C. y C. Ortiz (2008), "Instituciones, actores y mercados en el cambio empresarial. El caso de Cemex y Vitro”, México, D.F., UAM-Xochimilco.

Guillén, M. (2000), "Business groups in emerging economies: a resource-based view", Academy of Management Journal, vol. 43, $\mathrm{N}^{\mathrm{o}} 3$, junio, Briarcliff Manor, Academy of Management.
Hober, G. y G. Phillips (2008), "Real and financial industry booms and busts", NBER Working Papers Series, No 14290, Cambridge, Massachusetts, National Bureau of Economic Research, agosto [en línea] http://www.nber.org/papers/w14290.

Huerta, P. y J.E. Navas (2007), "Análisis de la relación entre la diversificación y los resultados empresariales: una visión teórica", Análisis económico, vol. 22, N 49, Azcapotzalco, D.F., Universidad Autónoma Metropolitana (UAM), enero-abril.

Khanna, T. e Y. Yafeh (2007), "Business groups in emerging markets: paragons or parasites?", Journal of Economic Literature, vol. 45, Pittsburgh, American Economic Association Publications, julio.

Levanti, C. (2001), "Prácticas empresariales y apertura económica en México", Comercio exterior, vol. 67, No 12, México, D.F., Bancomext, diciembre.

Mahmood, I. y W. Mitchell (2004), "Two faces: effects of business groups on innovation in emerging economies", Management Science, vol. 50, No 10, Hanover, Informs, octubre.

Morck, R. y M. Nakamura (2007), "Business groups and the big push: Meiji Japan's mass privatization and subsequent growth", NBER Working Papers Series, № 13171, Cambridge, Massachusetts, National Bureau of Economic Research, junio [en línea] http://www.nber.org/papers/w13171.

Mortimore, M. y W. Peres (2001), "La competitividad empresarial en América Latina y el Caribe", Revista de la CEPAL, $\mathrm{N}^{\circ} 74$ (LC/G.2135-P), Santiago de Chile, Comisión Económica para América Latina y el Caribe (CEPAL), agosto.

Pérez, J.A. y F. Vela (2008), "Cambio en la concentración industrial manufacturera en el contexto de apertura comercial de México, 1980-2003", Análisis económico, vol. 23, № 52, Azcapotzalco, D.F., Universidad Autónoma Metropolitana (UAM), eneroabril.

Rendón, A. (1997), “Grupos económicos en la década de los ochenta. Estrategia de diversificación o especialización”, Economía: teoría y práctica, $\mathrm{N}^{\circ}$ 8, México, D.F., UAM Xochimilco, enero-junio.

Rendón, A. y A. Morales (2008a), "Grupos económicos en la industria de alimentos: la estrategia de Gruma", Argumentos, $\mathrm{N}^{\circ}$ 57, México, D.F., Universidad Autónoma Metropolitana (UAM), mayo-agosto.

(2008b), "Estrategias de competencia (1987-2005)",Ide@s CONCYTEG, año 3, $\mathrm{N}^{\circ}$ 41, Guanajuato, México, Consejo de Ciencia y Tecnología del Estado de Guanajuato (CONCYTEG), noviembre.

Salas-Porras, A. (2006a), "Los grupos mexicanos y coreanos ante el desmantelamiento del Estado", documento presentado en el $52^{\circ}$ Congreso Internacional de Americanistas (Sevilla, España, 17 al 21 de julio de 2006).

(2006b), "Fuerzas centrípetas y centrífugas en red corporativa mexicana (1981-2001)", Revista mexicana de sociología, vol. 68, $\mathrm{N}^{\circ}$ 2, México, D.F., Instituto de Investigaciones Sociales, Universidad Nacional Autónoma de México, abril-junio.

Santiso, J. (2008), "La emergencia de las multilatinas", Revista de la CEPAL, No 95 (LC/G.2382-P), Santiago de Chile, Comisión Económica para América Latina y el Caribe (CEPAL), agosto.

Tarziján, J. y R. Paredes (2006), Organización industrial para la estrategia empresarial, México, D.F., Pearson Education.

Vázquez, M.A. (2004), "Grupos económicos en el norte de México", Problemas del desarrollo, vol. 35, No 137, México, D.F., Instituto de Investigaciones Económicas, Universidad Nacional Autónoma de México, abril-junio.

(1997), "Reestructuración económica y grupos empresariales en el norte de México", Economía y sociedad, No 3, Ciudad Juárez, Universidad Autónoma de Ciudad Juárez, agosto. 